\title{
Beryllium anomalies in solar-type field stars ${ }^{\star}$
}

\author{
N. C. Santos ${ }^{1,2}$, G. Israelian ${ }^{3}$, S. Randich ${ }^{4}$, R. J. García López ${ }^{3,5}$, and R. Rebolo 3,6 \\ ${ }^{1}$ Centro de Astronomia e Astrofísica da Universidade de Lisboa, Observatório Astronómico de Lisboa, Tapada da Ajuda, \\ 1349-018 Lisboa, Portugal \\ e-mail: nuno.santos@oal.ul.pt \\ 2 Observatoire de Genève, 51 Ch. des Maillettes, 1290 Sauverny, Switzerland \\ 3 Instituto de Astrofísica de Canarias, 38200 La Laguna, Tenerife, Spain \\ ${ }^{4}$ INAF/Osservatorio Astrofisico di Arcetri, Largo Fermi 5, 50125 Firenze, Italy \\ 5 Departamento de Astrofísica, Universidad de La Laguna, Av. Astrofísico Francisco Sánchez s/n, 38206, La Laguna, \\ Tenerife, Spain \\ ${ }^{6}$ Consejo Superior de Investigaciones Científicas, Spain
}

\section{Received 24 March 2004 / Accepted 11 June 2004}

\begin{abstract}
We present a study of beryllium (Be) abundances in a large sample of field solar-type dwarfs and sub-giants spanning a large range of effective temperatures. The Be abundances, computed using a very uniform set of stellar parameters and near-UV spectra obtained with 3 different instruments, are used to study the depletion of this light element. The analysis shows that $\mathrm{Be}$ is severely depleted for $\mathrm{F}$ stars, as expected by the light-element depletion models. However, we also show that beryllium abundances decrease with decreasing temperature for stars cooler than $\sim 6000 \mathrm{~K}$, a result that cannot be explained by current theoretical models including rotational mixing, but that is, at least in part, expected from the models that take into account internal wave physics. In particular, the light element abundances of the coolest and youngest stars in our sample suggest that $\mathrm{Be}$, as well as lithium ( $\mathrm{Li}$ ), has already been burned early during their evolution. Furthermore, we find strong evidence for the existence of a Be-gap for solar-temperature stars. The analysis of $\mathrm{Li}$ and Be abundances in the sub-giants of our sample also shows the presence of one case that has still detectable amounts of $\mathrm{Li}$, while $\mathrm{Be}$ is severely depleted. Finally, we compare the derived Be abundances with $\mathrm{Li}$ abundances derived using the same set of stellar parameters. This gives us the possibility to explore the temperatures for which the onset of $\mathrm{Li}$ and Be depletion occurs.
\end{abstract}

Key words. stars: abundances - stars: fundamental parameters

\section{Introduction}

The light elements lithium ( $\mathrm{Li}$ ), beryllium (Be), and boron (B) are important tracers of the internal stellar structure and kinematics. Since they are destroyed at relatively low temperatures, they give us an idea about how the material inside stars is mixed with the hotter interior. Their analysis, together or separately, can thus give us important information about the mixing and depletion processes.

Most studies of light elements in solar-type stars have been based on Li abundances. This is mostly because $\mathrm{Li}$ features are easier to measure from high-resolution optical spectra (e.g. the

\footnotetext{
* Based on observations collected with the VLT/UT2 Kueyen telescope (Paranal Observatory, ESO, Chile) using the UVES spectrograph (Observing runs 66.C-0116 A, 66.D-0284 A, and 68.C-0058 A), and with the William Herschel and Nordic Optical Telescopes, operated at the island of La Palma by the Isaac Newton Group and jointly by Denmark, Finland, Iceland, and Norway, respectively, in the Spanish Observatorio del Roque de los Muchachos of the Instituto de Astrofísica de Canarias.
}

Li6708 A line). On the other hand, the only available Be features (Be II) are located near the atmospheric near-UV cutoff. Using ground-based telescopes to measure $\mathrm{Be}$ abundances is thus not a simple task. The situation for B is even worst, and only with instruments in space is it possible to obtain the abundances for this element. It is important, however, to complement the $\mathrm{Li}$ studies with analysis of $\mathrm{Be}$ and (if possible) B in solar-type stars. These three elements are destroyed by (p, $\alpha$ )-reactions at different temperatures (about 2.5, 3.5, and $5.0 \times 10^{6} \mathrm{~K}$, for $\mathrm{Li}, \mathrm{Be}$ and $\mathrm{B}$, respectively). Their abundances can thus help us to probe different regions (depths) inside a solar-type star.

Beryllium is produced by spallation reactions in the interstellar medium, while it is burned in the hot stellar furnaces (e.g., Reeves 1994; Ramaty et al. 1997). Since it is burned at much higher temperatures than $\mathrm{Li}, \mathrm{Be}$ is depleted at lower rates, and thus we can expect to measure Be abundances in stars which have no detectable $\mathrm{Li}$ in their atmospheres (like intermediate-age late G- or K-type dwarfs). In fact, for about $50 \%$ of the known planet host stars no Li was 
detected (e.g., Israelian et al. 2004). Furthermore, Li studies have shown the presence of a significant scatter not only among field stars (Pasquini et al. 1994), but also among coeval stars in open clusters (Soderblom et al. 1993; García López et al. 1994; Pasquini et al. 1997; Randich et al. 1998; Jones et al. 1999). More specifically, a spread is seen among K-type stars in young clusters; this dispersion appears to be related to rotation or rotational history. A dispersion is also seen among solar-type stars in the solar age cluster M67; the reasons for this dispersion are still unknown. Also note that the dispersion is not observed for other old clusters (Randich et al. 2003). The presence of a dispersion at a given age can indeed complicate any study.

In a companion paper to this - Santos et al. (2004a), hereafter Paper I - we present a study of Be in a set of field stars known to harbor planetary mass companions, as well as in a comparison set of "single" stars. The main result of that paper is that the differences between the two groups of stars are very small, if any. In the current paper we use the same sample studied in Paper I to explore the Be depletion throughout the main-sequence and sub-giant branch. The results reveal some interesting and unexpected features. First, we confirm former results (Santos et al. 2002a) supporting that lower-temperature dwarfs burn Be more easily than their higher temperature counterparts. Be abundances seem to follow a trend similar to the one found for Li. We further show that there seems to exist a Be-dip for some solar-temperature dwarfs, and report on some sub-giants that have no measurable $\mathrm{Be}$ in their atmospheres, though one of them has detectable amounts of Li. Finally, Li and Be abundances for the same stars are compared, giving us the possibility to determine the temperature at which the onset of $\mathrm{Li}$ and Be depletion occurs.

\section{The data, stellar parameters, and chemical analysis}

The data used in this paper has already been fully described in Paper I. We refer to this for more details on the spectrographs/telescopes used, $S / N$ and resolution of the spectra, and for data-reduction details.

Except for BD-103166 (Gonzalez et al. 2001), all the stellar parameters were taken from the fully spectroscopic uniform study of Santos et al. (2004b), or derived using the same techique. Given that these parameters have been obtained using the same technique for all our stars, this gives us an important guarantee of uniformity in the analysis.

The Be abundances were then derived by fitting the spectral region around the Be II lines at 3130.420 and $3131.065 \AA$. In practice, only the latter line was used to derive the Be abundances, given the severe line-blending in the region around $3130.42 \AA$; the bluest line was thus only used to check the consistency of the fit. The analysis was done assuming LTE, using the 2002 version of the code MOOG (Sneden 1973), and a grid of Kurucz (1993) ATLAS9 atmospheres. The line list used was described in García López \& Pérez de Taoro (1998). The final precision of the abundance results is of the order of $0.15 \mathrm{dex}$. The Be abundance we have obtained from an analysis of the Kurucz Solar Atlas (Kurucz et al. 1984) is 1.10 , close to the value of 1.15 obtained by
Chmielewski et al. (1975). Again, for more details we point the reader to Paper I.

Finally, the $\mathrm{Li}$ abundances are a revised version of the values derived in Israelian et al. (2004), and the values of $v \sin i$ were taken, in most of the cases, from a calibration of the CORALIE cross-correlation function (see appendix in Santos et al. 2002b). In the rest of this paper, the solar Li abundance was taken from Grevesse \& Sauval (1998), $\log N(\mathrm{Li})=1.10$.

In Tables 1 and 2 we present the final Be abundances, stellar parameters, $v \sin i$ values, and $\mathrm{Li}$ abundances.

\section{Be as a function of $T_{\text {eff }}$}

In Fig. 4 (upper panel) we plot the derived Be abundances as a function of effective temperature for our program stars. In the plot, the circles denote dwarfs, while sub-giants are denoted with crosses. The Sun is denoted by the usual solar symbol, assuming a Be abundance of 1.10 (see Sect. 2).

Sub-giants were defined as those stars that fall considerably above the Main-Sequence in the HR diagram of Fig. 5. There are 6 of those, namely HD 10647, HD 23249, HD 27442, HD 38529, HD 117176, and HD 168443. Except for this latter star, all the others have surface gravity values below $4.1 \mathrm{dex}$ (HD 168443 has a $\log g=4.22$ ), attesting to their evolutionary status.

The Be abundances for the sub-giants should be considered separately, since their effective temperatures may have changed considerably from the value they had when the stars were on the main-sequence; extra mixing may already have occurred, causing dilution and/or depletion of their light-element contents.

A look at Fig. 4 (upper panel) shows that the Be abundances have a maximum near $6100 \mathrm{~K}$. Very little, if any, Be depletion has occurred for stars of this effective temperature. A similar maximum for the Li abundances is found at about the same effective temperature (see e.g. Fig. 6). For higher temperatures, the values of $\log N(\mathrm{Be})$ decrease with increasing temperature, forming the well known Be gap for F stars (e.g., Boesgaard \& King 2002), a feature that has a counterpart for Li. For temperature values lower than $\sim 6100 \mathrm{~K}$, and except for a few outliers, the Be abundances also show a clear but less steep decreasing trend with decreasing temperature. This trend was already mentioned and discussed in Santos et al. (2002a), and is similar to that typically found for Li.

As discussed in Paper I, the maximum seen near $6100 \mathrm{~K}$ may be attributed e.g. to Galactic chemical evolution effects, since most of the stars in the temperature interval between 6000 and $6200 \mathrm{~K}$ are particularly metal-rich, and Galactic Be abundances are known to increase with the metallicity (Rebolo et al. 1988; Boesgaard \& King 1993; Molaro et al. 1997; Boesgaard et al. 1999). If so, and excluding these objects, Be abundances might form a plateau for temperatures between $\sim 6000$ and $5600 \mathrm{~K}$ (see also Sect. 7), decreasing with decreasing temperature for $T_{\text {eff }}$ below $\sim 5600 \mathrm{~K}$. The cause for the observed trend for the lower temperature dwarfs in our sample can hardly be attributed to any metallicity effect, however, since some very metal-rich stars are present at these low temperatures. 
Table 1. Derived Be abundances (BD and HD numbers up to 80000 ).

\begin{tabular}{|c|c|c|c|c|c|c|c|c|c|c|c|}
\hline Star & $\begin{array}{l}T_{\text {eff }} \\
{[\mathrm{K}]}\end{array}$ & $\begin{array}{l}\log g_{\text {spec }} \\
{\left[\mathrm{cm} \mathrm{s}^{-2}\right]}\end{array}$ & $\begin{array}{c}\xi_{\mathrm{t}} \\
{\left[\mathrm{km} \mathrm{s}^{-1}\right]}\end{array}$ & $\overline{[\mathrm{Fe} / \mathrm{H}]}$ & $\overline{l o g} N(\mathrm{Be})$ & $\overline{\sigma \sigma(\mathrm{Be})}$ & Instr. $^{\dagger}$ & $\overline{S O S / N}$ & $\begin{array}{c}v \sin i \\
{\left[\mathrm{~km} \mathrm{~s}^{-1}\right]}\end{array}$ & Source $^{\dagger \dagger}$ & $\overline{l \log N(\mathrm{Li})}$ \\
\hline Sun $^{\star}$ & 5777 & 4.44 & 1.00 & 0.00 & 1.10 & 0.05 & - & - & 1.9 & (e) & 1.10 \\
\hline BD-103166 & 5320 & 4.38 & 0.85 & 0.33 & $<0.50$ & - & [2] & 20 & 1.58 & (a) & - \\
\hline HD 870 & 5447 & 4.57 & 1.13 & -0.07 & 0.80 & 0.15 & [1] & 130 & 1.77 & (a) & $<0.20$ \\
\hline HD 1461 & 5768 & 4.37 & 1.27 & 0.17 & 1.14 & 0.13 & [1] & 120 & 1.71 & (a) & $<0.51$ \\
\hline HD 1581 & 5956 & 4.39 & 1.07 & -0.14 & 1.15 & 0.11 & [1] & 140 & 2.16 & (a) & 2.37 \\
\hline HD 3823 & 5948 & 4.06 & 1.17 & -0.25 & 1.02 & 0.12 & [1] & 130 & 1.99 & (a) & 2.41 \\
\hline HD 4391 & 5878 & 4.74 & 1.13 & -0.03 & 0.64 & 0.11 & [3] & 150 & 2.72 & (a) & $<1.09$ \\
\hline HD 6434 & 5835 & 4.60 & 1.53 & -0.52 & 1.08 & 0.10 & [3] & 150 & 1.30 & (a) & $<0.85$ \\
\hline HD 7570 & 6140 & 4.39 & 1.50 & 0.18 & 1.17 & 0.10 & [3] & 180 & 3.82 & (a) & 2.91 \\
\hline HD 9826 & 6212 & 4.26 & 1.69 & 0.13 & 1.05 & 0.13 & [6] & 120 & 9 & (b) & 2.55 \\
\hline HD 10647 & 6143 & 4.48 & 1.40 & -0.03 & 1.19 & 0.10 & [3] & 150 & 4.87 & (a) & 2.80 \\
\hline HD 10697 & 5641 & 4.05 & 1.13 & 0.14 & 1.31 & 0.13 & [5] & 40 & - & - & 1.96 \\
\hline HD 10700 & 5344 & 4.57 & 0.91 & -0.52 & 0.83 & 0.11 & [3] & 180 & 0.90 & (a) & $<0.41$ \\
\hline HD 12661 & 5702 & 4.33 & 1.05 & 0.36 & 1.13 & 0.13 & [5] & 40 & - & - & $<0.98$ \\
\hline HD 13445 & 5163 & 4.52 & 0.72 & -0.24 & $<0.40$ & - & [1] & 150 & 1.27 & (a) & $<-0.12$ \\
\hline HD 14412 & 5368 & 4.55 & 0.88 & -0.47 & 0.80 & 0.11 & [3] & 190 & 1.42 & (a) & $<0.44$ \\
\hline HD 16141 & 5801 & 4.22 & 1.34 & 0.15 & 1.17 & 0.13 & [1] & 120 & 1.95 & (a) & 1.11 \\
\hline HD 17051 & 6252 & 4.61 & 1.18 & 0.26 & 1.03 & 0.13 & [1] & 150 & 5.38 & (a) & 2.66 \\
\hline HD 19994 & 6190 & 4.19 & 1.54 & 0.24 & 0.93 & 0.12 & [3] & 140 & 8.10 & (a) & 1.99 \\
\hline HD 20010 & 6275 & 4.40 & 2.41 & -0.19 & 1.01 & 0.10 & [3] & 180 & 4.63 & (a) & 2.13 \\
\hline HD 20766 & 5733 & 4.55 & 1.09 & -0.21 & $<-0.09$ & - & [3] & 200 & 1.98 & (a) & $<0.97$ \\
\hline HD 20794 & 5444 & 4.47 & 0.98 & -0.38 & 0.91 & 0.11 & [3] & 250 & 0.52 & (a) & $<0.52$ \\
\hline HD 20807 & 5843 & 4.47 & 1.17 & -0.23 & 0.36 & 0.11 & [3] & 160 & 1.74 & (a) & $<1.07$ \\
\hline HD 22049 & 5073 & 4.43 & 1.05 & -0.13 & 0.80 & 0.13 & [5] & 100 & 2.13 & (a) & $<0.25$ \\
\hline HD 22049 & 5073 & 4.43 & 1.05 & -0.13 & 0.75 & 0.31 & [3] & 200 & 2.13 & (a) & $<0.25$ \\
\hline HD 22049 & 5073 & 4.43 & 1.05 & -0.13 & 0.77 & - & avg & - & 2.13 & (a) & $<0.25$ \\
\hline HD 23249 & 5074 & 3.77 & 1.08 & 0.13 & $<0.15$ & - & {$[5]$} & 80 & 1.01 & (a) & 1.24 \\
\hline HD 23484 & 5176 & 4.41 & 1.03 & 0.06 & $<0.70$ & - & [3] & 140 & 2.40 & (a) & $<0.40$ \\
\hline HD 26965 & 5126 & 4.51 & 0.60 & -0.31 & 0.76 & 0.13 & {$[5]$} & 55 & 0.77 & (a) & $<0.17$ \\
\hline HD 27442 & 4825 & 3.55 & 1.18 & 0.39 & $<0.30$ & - & [3] & 110 & $\sim 0$ & (a) & $<-0.47$ \\
\hline HD 30495 & 5868 & 4.55 & 1.24 & 0.02 & 1.16 & 0.11 & [3] & 140 & 3.04 & (a) & 2.44 \\
\hline HD 36435 & 5479 & 4.61 & 1.12 & 0.00 & 0.99 & 0.12 & [3] & 210 & 4.58 & (a) & 1.67 \\
\hline HD 38529 & 5674 & 3.94 & 1.38 & 0.40 & $<-0.10$ & - & [2] & 60 & - & - & $<0.61$ \\
\hline HD 38858 & 5752 & 4.53 & 1.26 & -0.23 & 1.02 & 0.11 & [3] & 150 & 0.99 & (a) & 1.64 \\
\hline HD 43162 & 5633 & 4.48 & 1.24 & -0.01 & 1.08 & 0.11 & [3] & 160 & 5.49 & (a) & 2.34 \\
\hline HD 43834 & 5594 & 4.41 & 1.05 & 0.10 & 0.94 & 0.11 & [3] & 220 & 1.44 & (a) & 2.30 \\
\hline HD 46375 & 5268 & 4.41 & 0.97 & 0.20 & $<0.80$ & - & [3] & 90 & - & - & $<-0.02$ \\
\hline HD 52265 & 6103 & 4.28 & 1.36 & 0.23 & 1.25 & 0.11 & [1] & 120 & 3.95 & (a) & 2.88 \\
\hline HD 69830 & 5410 & 4.38 & 0.89 & -0.03 & 0.79 & 0.11 & [3] & 100 & 0.75 & (a) & $<0.47$ \\
\hline HD 72673 & 5242 & 4.50 & 0.69 & -0.37 & 0.70 & 0.13 & [3] & 180 & 1.19 & (a) & $<0.48$ \\
\hline HD 74576 & 5000 & 4.55 & 1.07 & -0.03 & 0.70 & 0.31 & [3] & 120 & 3.56 & (a) & 1.72 \\
\hline HD 75289 & 6143 & 4.42 & 1.53 & 0.28 & 1.38 & 0.10 & [2] & 30 & 3.81 & (a) & 2.85 \\
\hline HD 75289 & 6143 & 4.42 & 1.53 & 0.28 & 1.33 & 0.12 & [1] & 110 & 3.81 & (a) & 2.85 \\
\hline HD 75289 & 6143 & 4.42 & 1.53 & 0.28 & 1.36 & - & avg & 110 & 3.81 & (a) & 2.85 \\
\hline HD 76151 & 5803 & 4.50 & 1.02 & 0.14 & 1.02 & 0.11 & [3] & 110 & 1.02 & (a) & 1.88 \\
\hline
\end{tabular}

$\dagger$ The instruments used to obtain the spectra were: [1] UVES(A); [2] UVES(B); [3] UVES(C); [4] IACUB(A); [5] IACUB(B); [6] UES.

$\dagger \dagger$ The sources of the $v \sin i$ are: (a) CORALIE (Santos et al. 2002b); (b) Gonzalez (1997); (c) Gonzalez (1998); (d) Naef et al. (2004); (e) Soderblom (1982).

* The solar Be abundance was computed using the Kurucz Solar Atlas (Kurucz et al. 1984); the solar Li abundance was taken from Grevesse \& Sauval (1998). 
Table 2. Derived Be abundances (HD numbers from 80000 on).

\begin{tabular}{|c|c|c|c|c|c|c|c|c|c|c|c|}
\hline Star & $\begin{array}{l}T_{\text {eff }} \\
{[\mathrm{K}]}\end{array}$ & $\begin{array}{l}\log g_{\text {spec }} \\
{\left[\mathrm{cm} \mathrm{s}^{-2}\right]}\end{array}$ & $\begin{array}{c}\xi_{\mathrm{t}} \\
{\left[\mathrm{km} \mathrm{s}^{-1}\right]}\end{array}$ & {$[\mathrm{Fe} / \mathrm{H}]$} & $\log N(\mathrm{Be})$ & $\sigma(\mathrm{Be})$ & Instr. $^{\dagger}$ & $S / N$ & $\begin{array}{c}v \sin i \\
{\left[\mathrm{~km} \mathrm{~s}^{-1}\right]}\end{array}$ & Source $^{\dagger \dagger}$ & $\log N(\mathrm{Li})$ \\
\hline HD 82943 & 6016 & 4.46 & 1.13 & 0.30 & 1.37 & 0.17 & [4] & 20 & 1.65 & (a) & 2.51 \\
\hline HD 82943 & 6016 & 4.46 & 1.13 & 0.30 & 1.27 & 0.12 & [2] & 35 & 1.65 & (a) & 2.51 \\
\hline HD 82943 & 6016 & 4.46 & 1.13 & 0.30 & 1.27 & 0.12 & [1] & 140 & 1.65 & (a) & 2.51 \\
\hline HD $82943^{\star \star}$ & 6016 & 4.46 & 1.13 & 0.30 & 1.27 & - & avg & 140 & 1.65 & (a) & 2.51 \\
\hline HD 83443 & 5454 & 4.33 & 1.08 & 0.35 & $<0.70$ & - & [3] & 100 & 1.38 & (a) & $<0.52$ \\
\hline HD 84117 & 6167 & 4.35 & 1.42 & -0.03 & 1.11 & 0.11 & [3] & 160 & 4.85 & (a) & 2.64 \\
\hline HD 92788 & 5821 & 4.45 & 1.16 & 0.32 & 1.19 & 0.11 & [2] & 40 & 1.78 & (a) & 1.34 \\
\hline HD 95128 & 5954 & 4.44 & 1.30 & 0.06 & 1.23 & 0.11 & [4] & 100 & 2.1 & (c) & 1.83 \\
\hline HD 108147 & 6248 & 4.49 & 1.35 & 0.20 & 0.99 & 0.10 & [2] & 60 & 5.34 & (a) & 2.33 \\
\hline HD 114762 & 5884 & 4.22 & 1.31 & -0.70 & 0.82 & 0.11 & [4] & 65 & 1.5 & (c) & 2.20 \\
\hline HD 117176 & 5560 & 4.07 & 1.18 & -0.06 & 0.86 & 0.13 & [4] & 70 & 0.5 & (c) & 1.88 \\
\hline HD 120136 & 6339 & 4.19 & 1.70 & 0.23 & $<0.25$ & - & [6] & 90 & 14.5 & (b) & - \\
\hline HD 121504 & 6075 & 4.64 & 1.31 & 0.16 & 1.33 & 0.11 & [2] & 45 & 2.56 & (a) & 2.65 \\
\hline HD 130322 & 5392 & 4.48 & 0.85 & 0.03 & 0.95 & 0.13 & [4] & 35 & 1.47 & (a) & $<0.13$ \\
\hline HD 134987 & 5776 & 4.36 & 1.09 & 0.30 & 1.22 & 0.11 & [2] & 60 & 2.22 & (a) & $<0.74$ \\
\hline HD 143761 & 5853 & 4.41 & 1.35 & -0.21 & 1.11 & 0.12 & [6] & 120 & 1.5 & (c) & 1.46 \\
\hline HD 145675 & 5311 & 4.42 & 0.92 & 0.43 & $<0.65$ & - & [4] & 65 & 1 & (d) & $<0.03$ \\
\hline HD 168443 & 5617 & 4.22 & 1.21 & 0.06 & 1.11 & 0.13 & [4] & 55 & 1.68 & (a) & $<0.78$ \\
\hline HD 169830 & 6299 & 4.10 & 1.42 & 0.21 & $<-0.40$ & - & [3] & 130 & 3.35 & (a) & $<1.16$ \\
\hline HD 179949 & 6260 & 4.43 & 1.41 & 0.22 & 1.08 & 0.10 & [3] & 100 & 6.10 & (a) & 2.65 \\
\hline HD 187123 & 5845 & 4.42 & 1.10 & 0.13 & 1.08 & 0.12 & [4] & 55 & 1.73 & (d) & 1.21 \\
\hline HD 189567 & 5765 & 4.52 & 1.22 & -0.23 & 1.06 & 0.10 & [3] & 160 & 1.29 & (a) & $<0.82$ \\
\hline HD 192263 & 4947 & 4.51 & 0.86 & -0.02 & $<0.90$ & - & [3] & 60 & 2.02 & (a) & $<-0.39$ \\
\hline HD 192310 & 5069 & 4.38 & 0.79 & -0.01 & $<0.60$ & - & [3] & 180 & 0.85 & (a) & $<0.20$ \\
\hline HD 195019 & 5842 & 4.32 & 1.27 & 0.08 & 1.15 & 0.12 & [4] & 50 & 1.73 & (a) & 1.47 \\
\hline HD 202206 & 5752 & 4.50 & 1.01 & 0.35 & 1.04 & 0.11 & [3] & 130 & 2.44 & (a) & 1.04 \\
\hline HD 209458 & 6117 & 4.48 & 1.40 & 0.02 & 1.24 & 0.11 & [3] & 150 & 3.65 & (a) & 2.70 \\
\hline HD 210277 & 5532 & 4.29 & 1.04 & 0.19 & 0.91 & 0.13 & [1] & 110 & 1.39 & (a) & $<0.30$ \\
\hline HD 211415 & 5890 & 4.51 & 1.12 & -0.17 & 1.12 & 0.10 & [3] & 190 & 1.84 & (a) & 1.92 \\
\hline HD 217014 & 5804 & 4.42 & 1.20 & 0.20 & 1.02 & 0.12 & [6] & 100 & 2.1 & (c) & 1.30 \\
\hline HD 217107 & 5646 & 4.31 & 1.06 & 0.37 & 0.96 & 0.13 & [1] & 120 & 1.37 & (a) & $<0.40$ \\
\hline HD 222335 & 5260 & 4.45 & 0.92 & -0.16 & 0.66 & 0.22 & [1] & 110 & 1.25 & (a) & $<0.31$ \\
\hline HD 222582 & 5843 & 4.45 & 1.03 & 0.05 & 1.14 & 0.11 & [3] & 125 & 1.75 & (a) & $<0.59$ \\
\hline
\end{tabular}

The instruments used to obtain the spectra were: [1] UVES(A); [2] UVES(B); [3] UVES(C); [4] IACUB(A); [5] IACUB(B); [6] UES.

${ }^{\dagger \dagger}$ The sources of the $v \sin i$ are: (a) CORALIE (Santos et al. 2002b); (b) Gonzalez (1997); (c) Gonzalez (1998); (d) Naef et al. (2004).

${ }^{\star \star}$ Given the lower $S / N$ of the IACUB spectrum, only the UVES spectra were considered.

Interestingly, this latter result is not confirmed by other studies of Be abundances in open clusters $\mathrm{F}$ and $\mathrm{G}$ dwarfs (e.g., Boesgaard \& King 2002; Boesgaard et al. 2003a,b). It should be mentioned, however, that in their studies these authors have not analyzed stars with temperatures much below $5500 \mathrm{~K}$. Furthermore, the stars studied by Boesgaard \& King (2002) and Boesgaard et al. (2003a,b) are considerably younger than the "average" field stars in our sample. The fact that we do observe a decrease of the Be abundances as a function of decreasing temperature can be seen as evidence that $\mathrm{Be}$ is burned during the main-sequence evolution of these stars.

In the lower panel of Fig. 4 we present the same plot but now we have superposed on the Be measurements a set of Yale beryllium depletion isochrones from Pinsonneault et al. (1990)
- dashed lines - for solar metallicity and an age of $1.7 \mathrm{Gyr}$ (to our knowledge there are no isochrones available for higher ages). Given that it is not possible to know what was the initial Be abundance for all the targets, we will assume an initial $\log N(\mathrm{Be})$ of 1.26 for all stars, i.e., between Solar (1.10 see Sect. 2) and meteoritic (1.42 - Anders \& Grevesse 1989).

For temperatures above roughly $5600 \mathrm{~K}$, the Pinsonneault et al. (1990) models seem to follow reasonably well the observed trends. This confirms the result of Stephens et al. (1997), who have already shown that these models (improved Yale models by Deliyannis \& Pinsonneault (1993) do not show a clear difference) are quite satisfactory for stars with $5600 \mathrm{~K} \leq$ $T_{\text {eff }} \leq 6500 \mathrm{~K}$. However, it is also clear from the figure that there seems to be a clear discrepancy between the model 


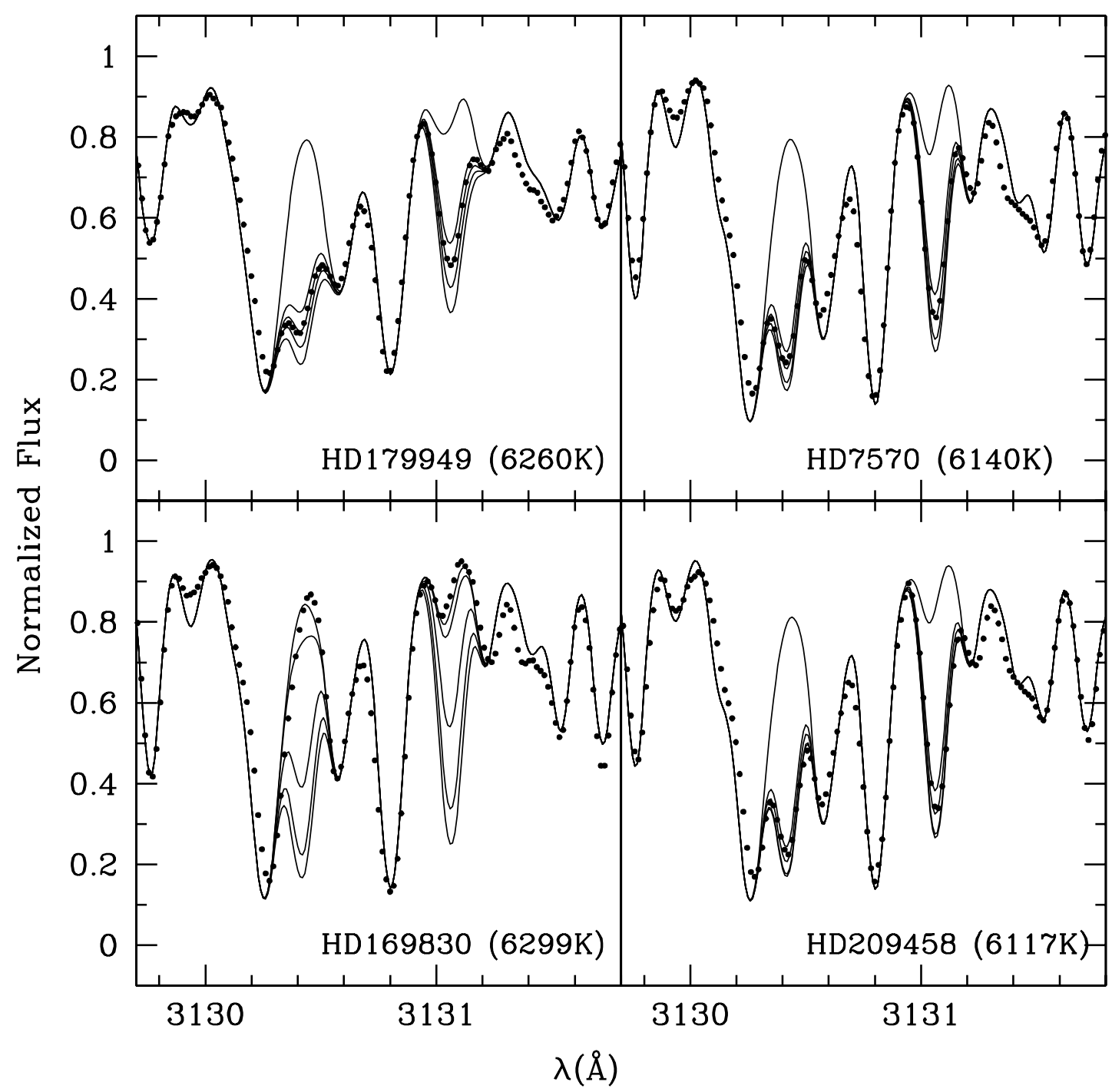

Fig. 1. Spectral syntheses (continuous lines) and observed spectra (points) in the Be II line region for 4 of the hottest dwarfs in our sample. In all panels the upper and lower syntheses were done with a $\log N(\mathrm{Be})$ of 1.42 (meteoritic) and -10.0 (essentially no Be), respectively. The three intermediate syntheses for all stars except HD 169830 correspond to the optimal fit and to fits with abundance variations of \pm 0.15 dex. For HD 169830, these correspond to abundances of $1.15,0.65$, and -0.35 dex. Stellar effective temperatures are also shown.

predictions and the observed abundances for lower temperature stars. As already noted in Santos et al. (2002a), while for these objects we observe a decrease in the Be abundance as a function of decreasing temperature, the model predictions go in the opposite sense.

There are several possibilities for explaining this observed inconsistency. One way would be to say that the derived abundances have some bias as a function of effective temperature. However, and as we can see from the plots of Fig. 3, our synthetic spectra seem to fit quite well the whole Be-line region for the lower temperature stars in our samples. Although we consider it quite unlikely that errors in the spectroscopic analysis are causing the whole observed trend, we should mention that the Be II line region is very crowded. García López et al. (1995) studied in detail the sensitivity of the observed feature at $\lambda 3131 \AA$ to Be abundance for low-mass stars. For effective temperatures below $\sim 5100 \mathrm{~K}$ the feature starts to be dominated by the contribution of another element (likely Mn I).
This makes it difficult to determine accurate Be abundances for the coolest stars of our sample.

As already pointed out in Paper I, the usefulness of the Be II line for deriving Be abundances will thus decrease with temperature to a point where it will be useless. However, the exact temperature at which this occurs will depend on the resolution and signal-to-noise ratio of the observed spectra. Even if the observed feature is not dominated by the Be transition, it is possible to obtain a reliable Be measurement by fitting synthetic spectra with different Be abundances, as long as the $S / N$ of the data is high enough.

In our case, the increasing dispersion observed for Be abundances of stars with temperature below $\sim 5400 \mathrm{~K}$ possibly reflects the increasing difficulty in deriving the Be abundances for the lowest temperature stars, although a real "physical" reason cannot be completely excluded. However, it should be mentioned that the decreasing trend of Be abundances as a function of decreasing $T_{\text {eff }}$ starts already for temperature values 


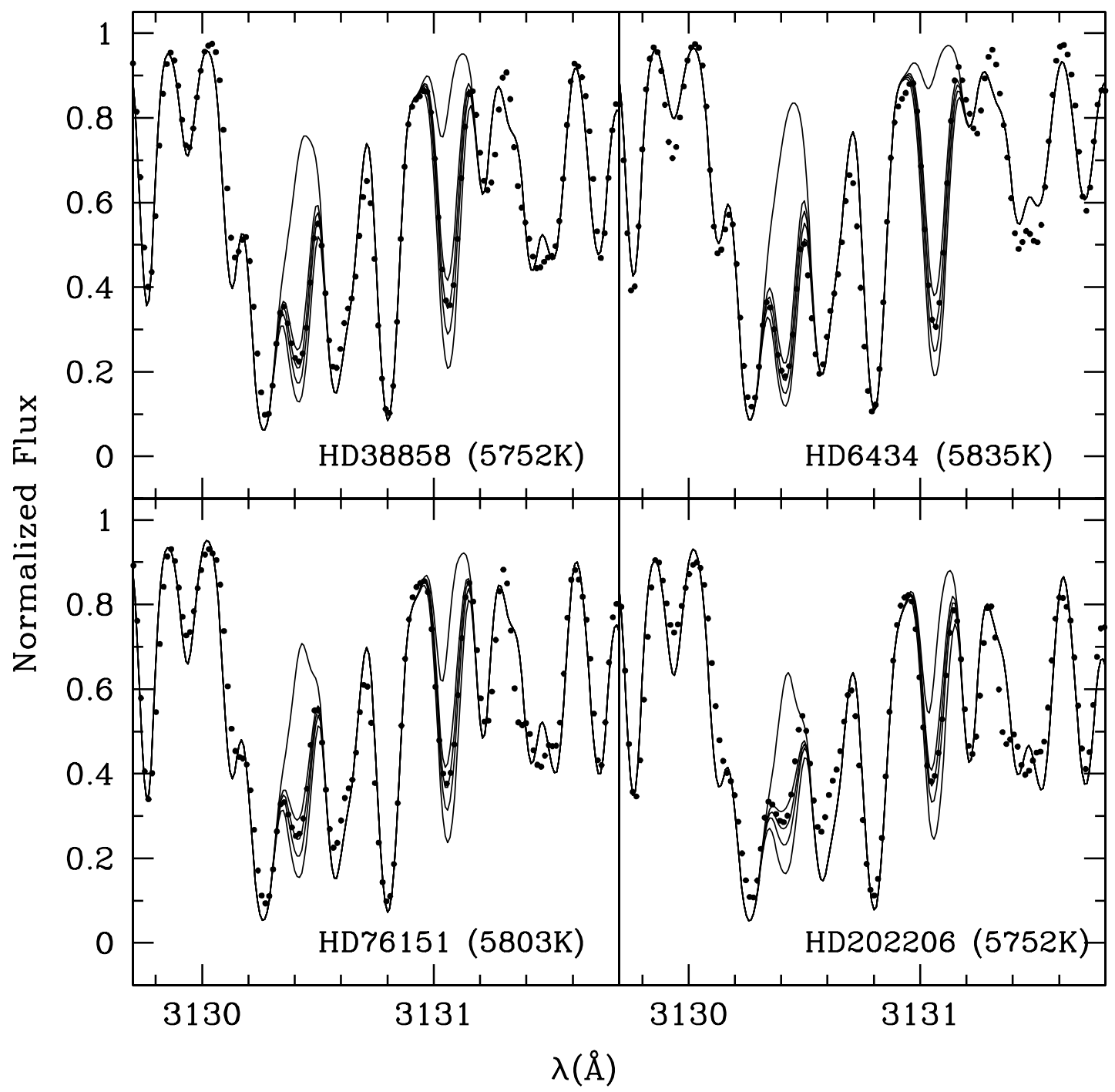

Fig. 2. Spectral syntheses (continuous lines) and observed spectra (points) in the Be II line region for 4 solar-temperature dwarfs in our sample. In all panels, the upper and lower syntheses were done with a $\log N(\mathrm{Be})$ of 1.42 (meteoritic) and -10.0 (essentially no Be), respectively. For all stars the three intermediate syntheses correspond to the optimal fit and to fits with abundance variations of \pm 0.15 dex.

only $\sim 150 \mathrm{~K}$ below the solar temperature (for which the line list was built). This makes very unlikely that the observed trend may be attributed to errors in the spectroscopic analysis only.

In this sense, one other possible source of error could arise from the presence of a "missing near-UV opacity", as discussed by Balachandran \& Bell (1998), something that we have not considered. However, the need to include this extra opacity is not established. Allende Prieto \& Lambert (2000), for example, have shown that it is possible to make a good fit of the solar spectrum without taking into account this extra-opacity. Furthermore, the problem discussed by Balachandran \& Bell (1998) refers to the solar case, and has not been discussed for other temperature regimes.

Another possibility for explaining the observed trend would be to consider that this has something to do with the presence of planets. Indeed, about one half of the stars in our sample are planet-hosts. If stellar pollution is very important among these objects, we could probably expect earlier-type dwarfs to present higher Be abundances, given that they have shallower convective envelopes. However, and as discussed in PaperI, there does not seem to be a large difference in the Be content of stars with and without known giant planets. We thus have no reason to believe that the presence of planets is causing the observed inconsistency. Curiously, Israelian et al. (2004) have shown that in the temperature interval between 5600 and $5850 \mathrm{~K}$ planet hosts are Li deficient when compared with field dwarfs. However, both samples do present the same Be trend as a function of $T_{\text {eff }}$ (see Paper I). In other words, the difference noticed for $\mathrm{Li}$ is aparentely not found for Be. We should mention, however, that the relatively low number of points may preclude a firmer conclusion.

Murray et al. (2001) have suggested that the stars in the solar neighborhood have accreted an average of $\sim 0.5 M_{\odot}$, a value compatible with the upper limit found for the Hyades cluster by Quillen (2002). This small amount of material, which would pollute more efficiently the hottest stars in our sample (these have shallower convective envelopes) is, however, probably not enough to explain the observed increasing trend. 


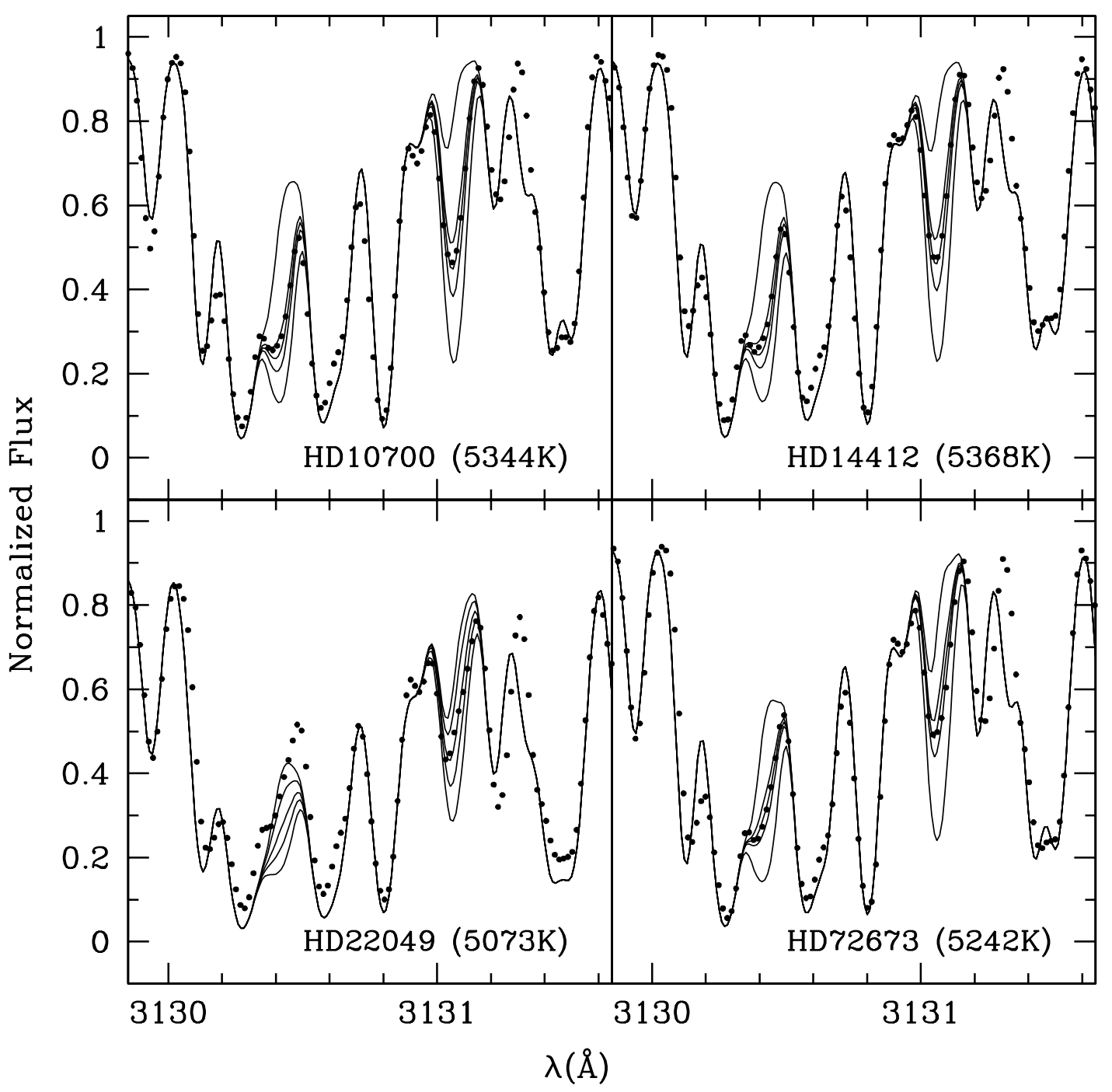

Fig. 3. Spectral syntheses (continuous lines) and observed spectra (points) in the Be II line region for 4 of the coolest dwarfs in our sample. In all panels, the upper and lower syntheses were done with a $\log N(\mathrm{Be})$ of 1.42 (meteoritic) and -10.0 (essentially no Be), respectively. For all stars expect HD 22049, the three intermediate syntheses correspond to the optimal fit and to fits with abundance variations of \pm 0.15 dex. For this star, the three intermediate fits were done using Be abundances of 1.05, 0.75 (best fit), and 0.25 dex.

It has been shown by Swenson et al. (1994) that even a small change in the oxygen abundance may have a strong effect on the Li depletion rate in solar-type stars. This effect is due to the change of the depth of the convective envelope induced by the change of the global opacity. Although we do not have oxygen abundances for our targets, they are spread over a wide range of metallicities. It is thus not very likely that the observed inconsistency is due to such an effect.

Interestingly, the models of Montalban \& Schatzman (2000), which include mixing by internal waves (dotted line ${ }^{1}$ in Fig. 4), could solve at least part of the problem, since they do predict an increasing Be depletion as a function of decreasing temperature (see also their Fig. 4). While this model still overestimates the Be abundances for the cooler stars in our sample, it fits our data much better than the Pinsonneault et al. (1990) models do.

\footnotetext{
1 The curve was taken directly from their Fig. 4.
}

\section{A Be gap for solar-temperature dwarfs}

In Fig. 6 (upper panel) we present the same plot of Fig. 4, but highlighting a small group of solar-temperature stars that seem to present abnormally low Be abundances, falling completely outside of the main trend. The spectral syntheses for four of these stars are presented in Fig. 7, and for the remaining in Fig. 8. These objects, all in the temperature regime between roughly $5600 \mathrm{~K}$ and $5900 \mathrm{~K}$, lie in some kind of Be-gap, similar to the one suggested to exist for $\mathrm{Li}$ in the same temperature regime (Pasquini et al. 1994; Chen et al. 2001).

To understand the situation it is important to check the nature of these particular stars. Four of them (HD 4391, HD 20766, HD 20807, and HD 114762) are dwarfs, while HD 38529 is one of the most evolved stars in our sample, being already in the sub-giant regime (see Tables 1 and 2). This latter case will be discussed in the next section.

As mentioned in Paper I, HD 4391, HD 20766, and HD 20807 are not known to harbor any planetary-mass 


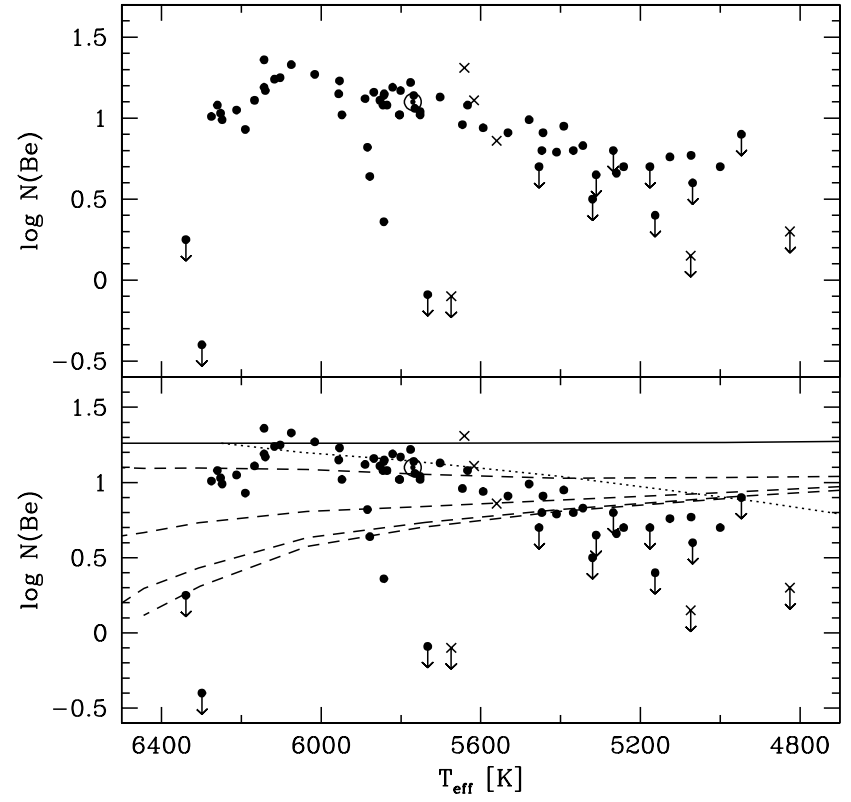

Fig. 4. Upper panel: derived Be abundances as a function of $T_{\text {eff. }}$ Dots represent dwarfs, while crosses denote sub-giants. The Sun is denoted by the usual symbol. Lower panel: same as above, but superposing the Be depletion models of Pinsonneault et al. (1990) (Case A) for solar metallicity and an age of $1.7 \mathrm{Gyr}$ (dashed lines). From top to bottom, the lines represent a standard model (solid line), and 4 models with different initial angular momentum (Tables 3 to 6 of Pinsonneault et al. 1990). The solid line represents the initial Be abundance of 1.26 (see text). The dotted line represents the Be depletion isochrone for $4.5 \mathrm{Gyr}$ taken from the models including mixing by internal waves of Montalban \& Schatzman (2000).

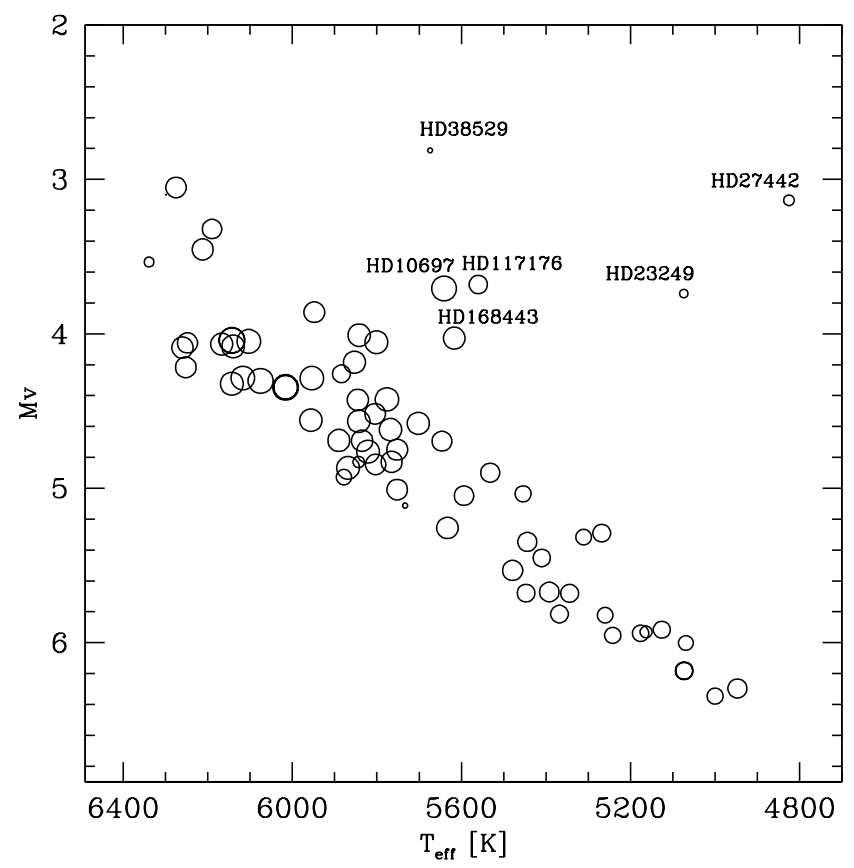

Fig. 5. HR diagram for the stars studied in this paper. The size of the circles is proportional to the Be abundance. Six of the stars (labeled) fall considerably outside the main-sequence, and are thus probably sub-giants. Absolute magnitudes were computed from Hipparcos parallaxes and $V$ magnitudes (ESA 1997). For clarity, no difference is made between stars with Be detections and with only an upper limit.

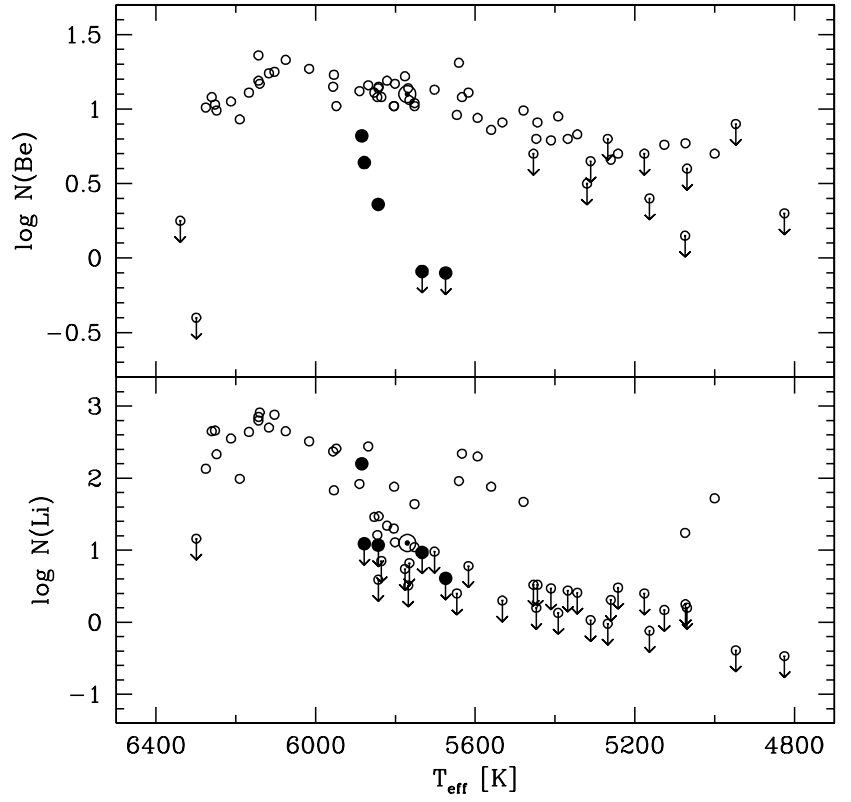

Fig. 6. Upper panel: Be abundances as a function of effective temperature for the stars in our sample. No distinction was made between dwarfs and sub-giants. The 5 stars ( 4 dwarfs and 1 sub-giant) with anomalous Be abundances are denoted by the filled circles (see text and Fig. 7 for more details). Lower panel: same as above, but for Li.

companion. HD 114762 and HD 38529, on the other hand, do have low mass companions in orbit.

We have estimated the ages of HD 4391, HD 20766, and HD 20807 from their chromospheric activity (derived from Ca II H and K lines - Vaughan et al. 1978). Values for the Mount-Wilson $S$ index were taken from Henry et al. (1996) and from an analysis of the CORALIE spectra, a process described in Santos et al. (2000). Given the similarity of the values, we have decided to take the average $S_{\mathrm{MW}}$ value for each star to compute the stellar age. This latter was derived from the calibration presented in Henry et al. (1996) (taken from Donahue 1993). The knowledge of the $S$ index also gives us the possibility to access the rotational period of the stars (Noyes et al. 1984). The results are summarized in Table 3.

An inspection of Table 3 shows that all these stars seem to have very similar ages, rotation periods, as well as projected rotational velocities. Furthermore, and as can be seen from Table 1, their metallicities are all slightly below solar. Actually, these three stars are not very different from many other objects listed in Tables 1 and 2, occupying the same temperature regime (including our Sun; good examples are HD 38858 and HD $189567^{2}$ ) but having "normal" Be abundances.

HD 4391, HD 20766, and HD 20807 only have upper-limit estimates for the $\mathrm{Li}$ abundance, making it difficult to compare the Li/Be ratios (see Fig. 6, lower panel).

For HD 114762, however, the Li abundance is quite high $(\log N(\mathrm{Li})=2.20)$, which poses the problem of explaining how this star has become fairly depleted in Be (by a factor of $\sim 4$, if we suppose that the original Be abundances was similar to the

2 These two stars also have similar or lower chromospheric activity levels, implying similar or higher ages. 


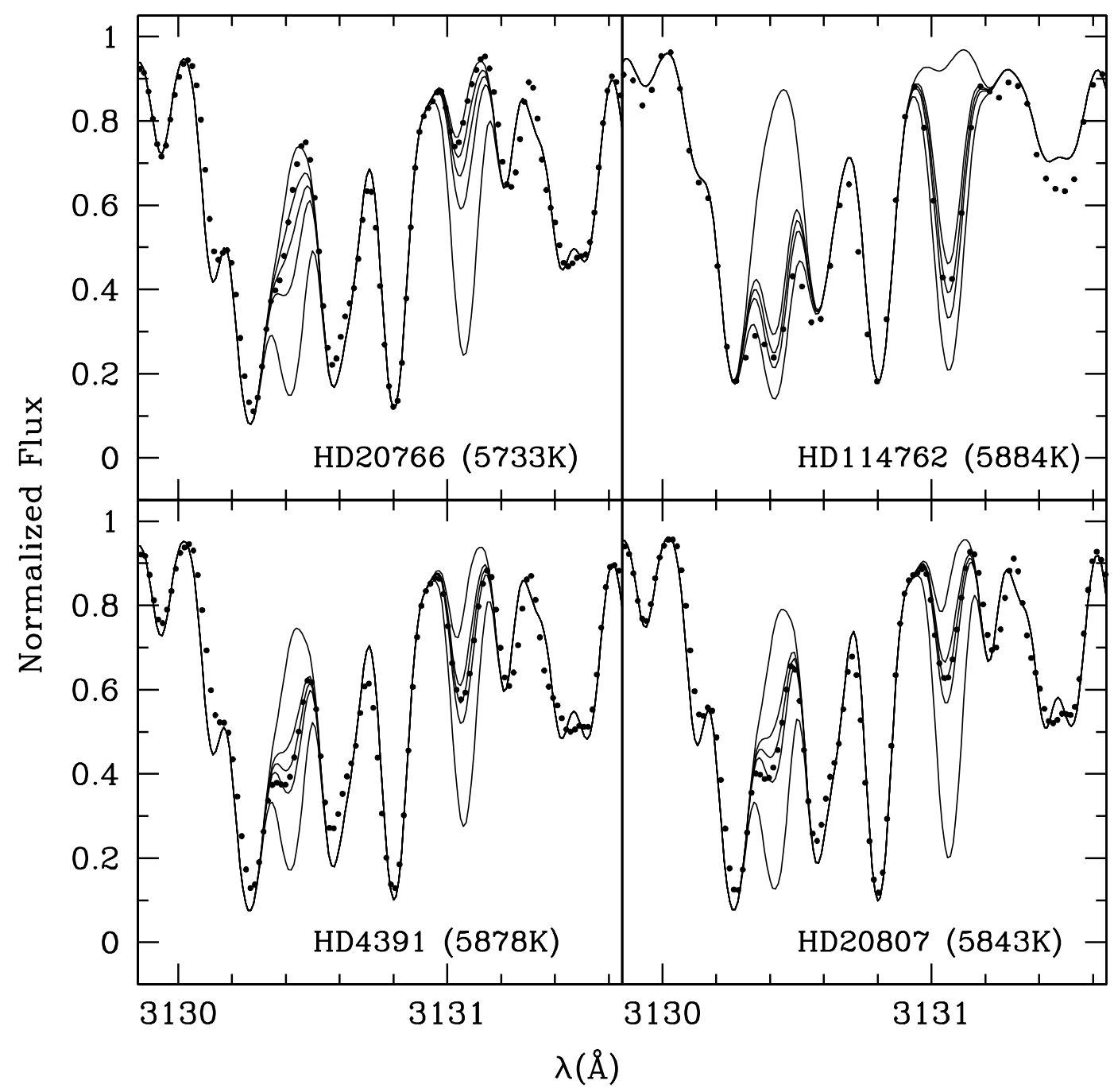

Fig. 7. Spectral syntheses (continuous lines) and observed spectra (points) in the Be II line region for four stars discussed in Sect. 4. In all panels, the upper and lower syntheses were done with a $\log N(\mathrm{Be})$ of 1.42 (meteoritic) and -10.0 (essentially no Be), respectively. The three intermediate syntheses for all but HD 20766 correspond to the optimal fit and to fits with abundance variations of \pm 0.15 dex. For this particular star, the three intermediate syntheses were done with Be abundances of $0.50,0.20$, and -0.10 .

one found in meteorites) while keeping so much Li in its atmosphere (compared to the meteoritic value of 3.31, Anders $\&$ Grevesse 1989, it is depleted by a factor of 12). However, one must take into account that HD 114762 is the most deficient star in our sample. Due to the chemical evolution of $\mathrm{Be}$ in the galaxy, this might imply that the original Be abundance was considerably lower than meteoritic for this object (e.g., Rebolo et al. 1988; Boesgaard \& King 1993; Molaro et al. 1997; Boesgaard et al. 1999) - see also García López et al. (in preparation). Indeed, its Be abundance is perfectly compatible with the one found for stars of its metallicity (e.g., Boesgaard et al. 1999).

In any case, the three remaining dwarfs (HD 4391, HD 20766, and HD 20807) clearly show that there seems to be an unexpected Be-gap for solar temperature stars, a region for which, at least in some cases, Be depletion is particularly severe. Some process capable of significantly increasing the mixing in the stellar interior seems to have played some role, and produced the observed scatter in the Be abundances. This process may be related to the possibility that different premain-sequence rotational histories may exist for different stars of similar mass, age, and metallicity (see e.g., Barnes 2003).

Finally, it is interesting to see that HD 20766 and HD 20807, two stars of the known wide visual binary system $\zeta^{1}$ and $\zeta^{2}$ Ret (with similar radial velocities and proper motions), are the two most Be-poor stars among the four objects discussed above. This fact could hint that a bona-fide explanation (although unlikely) for their very low Be abundances, is an abnormally low initial Be content. Curiously though, these stars have previously been noticed to be somehow abnormal (cf. Da Silva \& Foy 1987; Rocha-Pinto et al. 2002).

\section{Sub-giants}

Six stars in our sample have a position considerably above the HR diagram (see Fig. 5). These objects are probably sub-giants, stars that are already evolving out of the main sequence. During this process, the convective envelopes of the stars deepen, and 


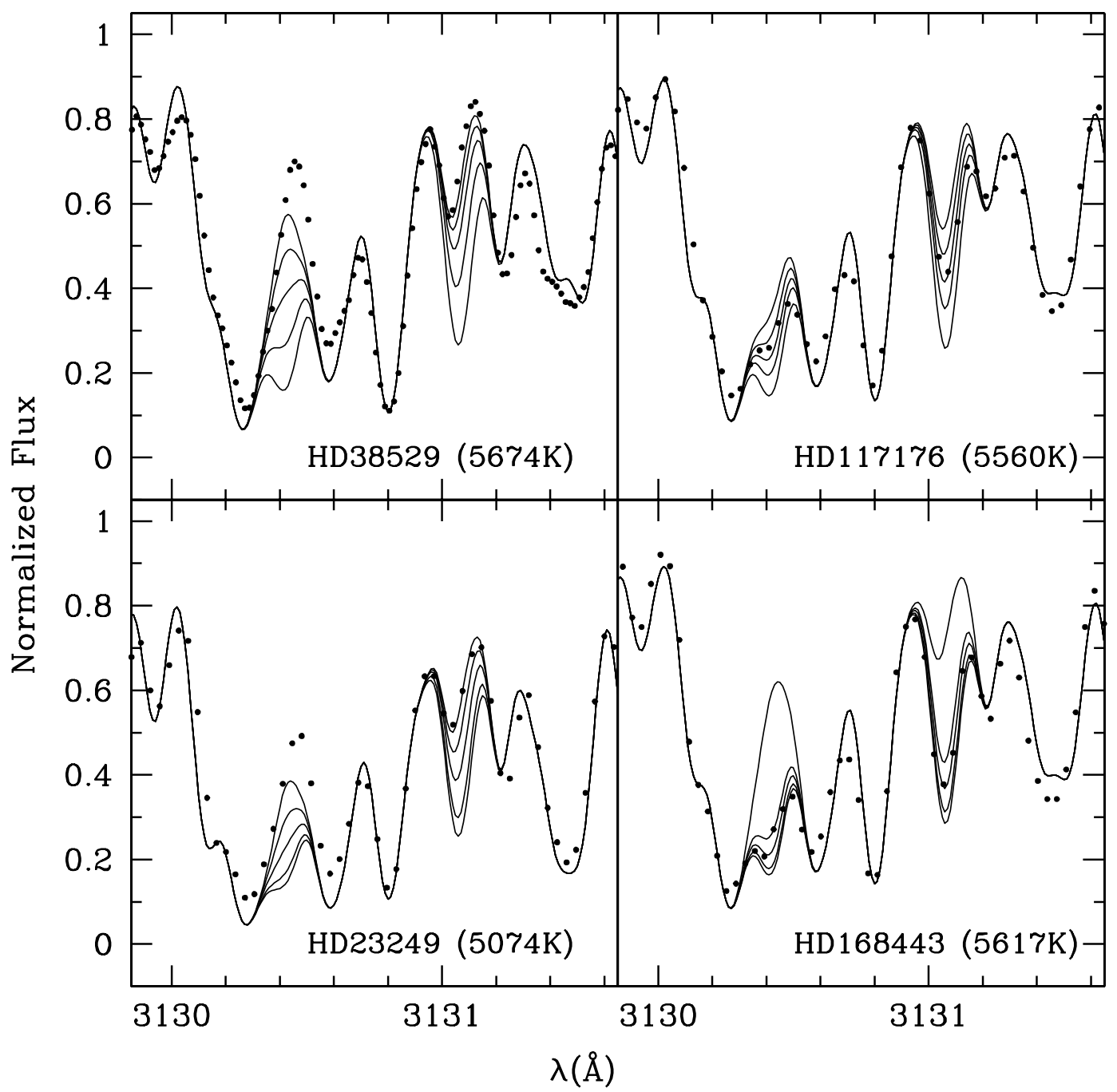

Fig. 8. Spectral syntheses (continuous lines) and observed spectra (points) in the Be II line region for four of the stars discussed in Sect. 5. In all panels, the upper and lower syntheses were done with a $\log N(\mathrm{Be})$ of 1.42 (meteoritic) and -10.0 (essentially no Be), respectively. The three other spectral syntheses for HD 23249 correspond to Be abundances of 1.15, 0.65, and 0.15 dex, while for HD 38529 these correspond abundances of $0.80,0.35$, and -0.10 . For HD 117176 and HD 168443 the three intermediate fits correspond to the optimal fit and to fits with abundance variations of $\pm 0.20 \mathrm{dex}$.

the light-element abundances can suffer considerable depletion/dilution. It is thus wise to treat these cases separately from the other stars (dwarfs).

In Fig. 9 we present a plot of the $\mathrm{Be}$ and $\mathrm{Li}$ abundances as a function of effective temperature, calling the attention to the position of the sub-giants in the plot (filled circles) - see also Fig. 8. As a general comment, these stars do follow the general trend of Be vs. $T_{\text {eff }}$ observed for dwarfs. Let us discuss these cases in more detail, separating these sub-giants into four groups, as follows.

HD 27442 and HD 38529. These two stars (both planethosts - Paper I, with $[\mathrm{Fe} / \mathrm{H}] \geq 0.21$ ), with very different temperatures, both have depleted their Li and Be abundances; only upper limits of these were derived, making it impossible to explore their $\mathrm{Li} / \mathrm{Be}$ ratios. HD 27442 is amongst the lowest temperature stars in our sample. Its position in the Be vs. $T_{\text {eff }}$ diagram follows the main trend. On the other hand, HD 38529 lies in the Be gap for solar-temperature stars discussed in the
Table 3. Data for HD 4391, HD 20766, and HD 20807 (see text for more details).

\begin{tabular}{lccccc}
\hline \hline Star & $S_{\mathrm{MW}}$ & $B-V^{c}$ & $\begin{array}{c}\text { Age } \\
(\mathrm{Gyr})\end{array}$ & $\begin{array}{c}P_{\text {rot }} \\
(\text { days })\end{array}$ & $\begin{array}{c}v \sin i^{d} \\
\left(\mathrm{~km} \mathrm{~s}^{-1}\right)\end{array}$ \\
\hline HD 4391 & $0.28^{a} / 0.26^{b}$ & 0.64 & 1.2 & 12 & 2.72 \\
HD 20766 & $0.23^{a} / 0.26^{b}$ & 0.64 & 1.6 & 14 & 1.98 \\
HD 20807 & $0.19^{a} / 0.23^{b}$ & 0.60 & 2.1 & 13 & 1.74 \\
\hline
\end{tabular}

${ }^{a}$ Taken from Henry et al. (1996).

${ }^{b}$ Derived using CORALIE spectra (Santos et al. 2000).

${ }^{c}$ Taken from the Hipparcos catalogue (ESA 1997).

${ }^{d}$ Derived from the width of the CORALIE cross-correlation function (Santos et al. 2002b).

previous section, suggesting that the same mechanism that was able to deplete $\mathrm{Be}$ in the dwarfs that are in this temperature interval may also have played some role here. Curiously, these 


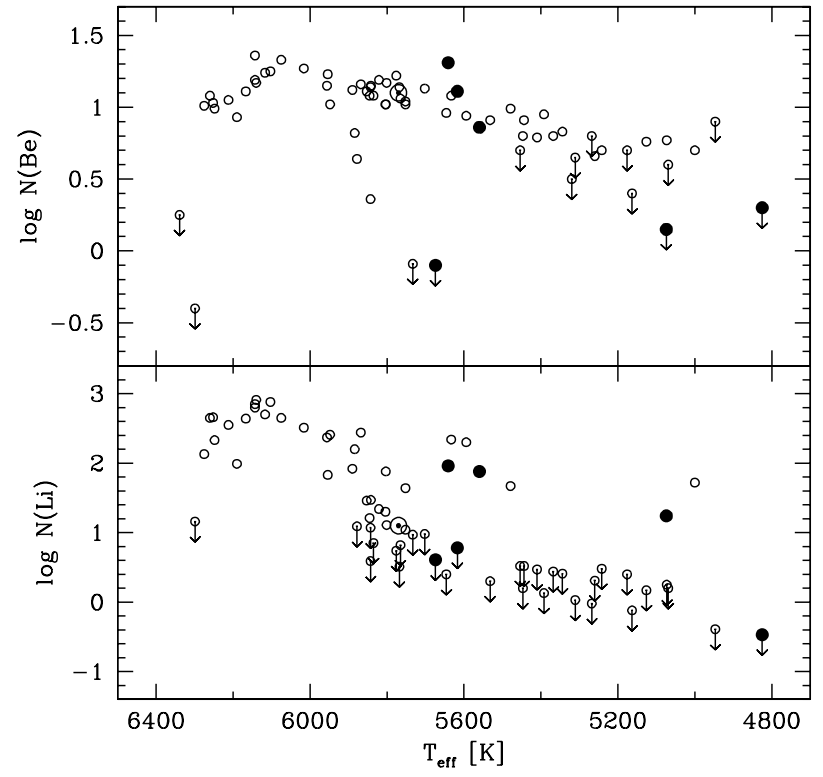

Fig. 9. Upper panel: Be abundances as a function of effective temperature for the stars in our sample. The 6 sub-giant stars are denoted by the filled circles. Lower panel: same as above, but for Li.

are the two sub-giants with the lowest absolute magnitude in the HR diagram of Fig. 5. Primas et al. (1998) have identified a sub-giant (HD 160617) in the same temperature regime as the objects discussed in Sect. 4 that is Li normal, but boron and possibly Be depleted. Given the low metallicity of this star $([\mathrm{Fe} / \mathrm{H}]=-1.8)$, however, the authors have proposed that the observed trend has to do with the low boron and Be abundances existent in the Galactic region where (and when) this star was formed. In general, none of the stars mentioned above seems to defy light-element depletion models, in the sense that their light-element depletion pattern is consistent with Li being burned faster than $\mathrm{Be}$.

HD 10697 and HD 117176. These stars, both planet-hosts, seem to have preserved both their $\mathrm{Li}$ and $\mathrm{Be}$ abundances. However, if on one hand they present "normal" Be abundance, their Li abundances are much higher than typically found for stars of their temperature. An extensive discussion of these two stars was already done in Santos et al. (2002a). The most convincing explanation found is that these two objects have just left the main sequence, where they were hotter than they are now, but that there has not yet been time for their $\mathrm{Li}$ (and $\mathrm{Be}$ ) to become strongly depleted. Indeed, a look at Fig. 9 shows that dwarfs that are merely $250 \mathrm{~K}$ hotter are able to maintain high levels of $\mathrm{Li}$ and $\mathrm{Be}$ in their atmospheres. Alternative explanations for the observed trend imply a scenario of planetary material engulfment, or that the $\mathrm{Li}$ and $\mathrm{Be}$ "excesses" observed are due to a dredge up effect from a "buffer" below the former main sequence convective envelope (Deliyannis et al. 1990). It should be mentioned, however, that this latter scenario does not seem to be supported by current observations (e.g., Randich et al. 1999). For a more thorough discussion we point the reader to Santos et al. (2002a).

$H D$ 168443. This sub-giant is probably the least evolved star amongst these 6 , as attested by it surface gravity value

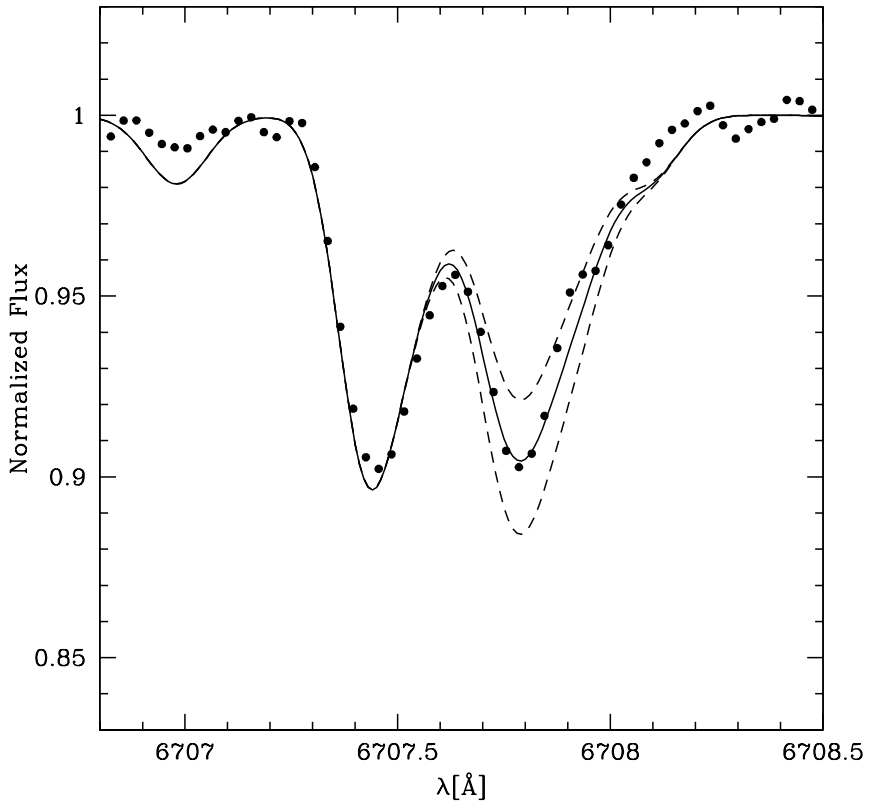

Fig. 10. Observed CORALIE spectrum of HD 23249 in the region near the Li6708 $̊$ line (points) together with 3 spectral syntheses for abundances of $\log N(\mathrm{Li})=1.14,1.24$ (best fit) and 1.34 dex. The spectral syntheses were done using the detailed line list used in Israelian et al. (2001). The small inconsistencies in the fit are probably due to the low temperature of this star, while the line list was optimized for the Sun. The strength of the Li line together with the quality of the line list used gives us a guarantee that the observed line is indeed due to Li and not any other element.

of $\log g=4.22$, and the relatively small height above the main sequence (see Fig. 5). Its position in both the $\mathrm{Li}$ and $\mathrm{Be}$ vs. $T_{\text {eff }}$ plots is that expected for stars of its temperature. Curiously, its position in the HR diagram of Fig. 5 is very similar to those of HD 10697 and HD 117176, although the three stars present quite different Be abundances.

HD 23249. This "single" star (not known to have any planetary companion), one of the coolest and most evolved $(\log g=$ 3.77) objects in our sample, is probably the most enigmatic case. Although its Be abundance is clearly low (only an upper limit was found - see also Fig 8), its Li content seems to be particularly high for its temperature, and a clear Li detection was possible for this star (see Fig. 10). Considering the upper limit derived for the $\mathrm{Be}$ abundance $\log N(\mathrm{Be})=0.15$, and the value of $\log N(\mathrm{Li})=1.14$, this star is depleted in Be by at least a factor of 13 (considering an initial Be abundance of 1.26 - see above), and its Li is depleted by a much larger factor of 150 , considering initially a meteoritic Li abundance of 3.31 (Anders $\&$ Grevesse 1989). For this case to be consistent with the expected light-element depletion pattern, the existence of $\mathrm{Li}$ in this cool (old) sub-giant leads us to suppose that a process similar to the one discussed above for HD 10697 and HD 117176 has occurred, which is more difficult in this case, since this star is much cooler. An alternative possibility to explain the observed abundances would be to consider that the Li "excess" observed is due to a dredge up effect from a "buffer" below the former main sequence convective envelope (Deliyannis et al. 1990; Randich et al. 1999), or to the accretion of metal-rich 


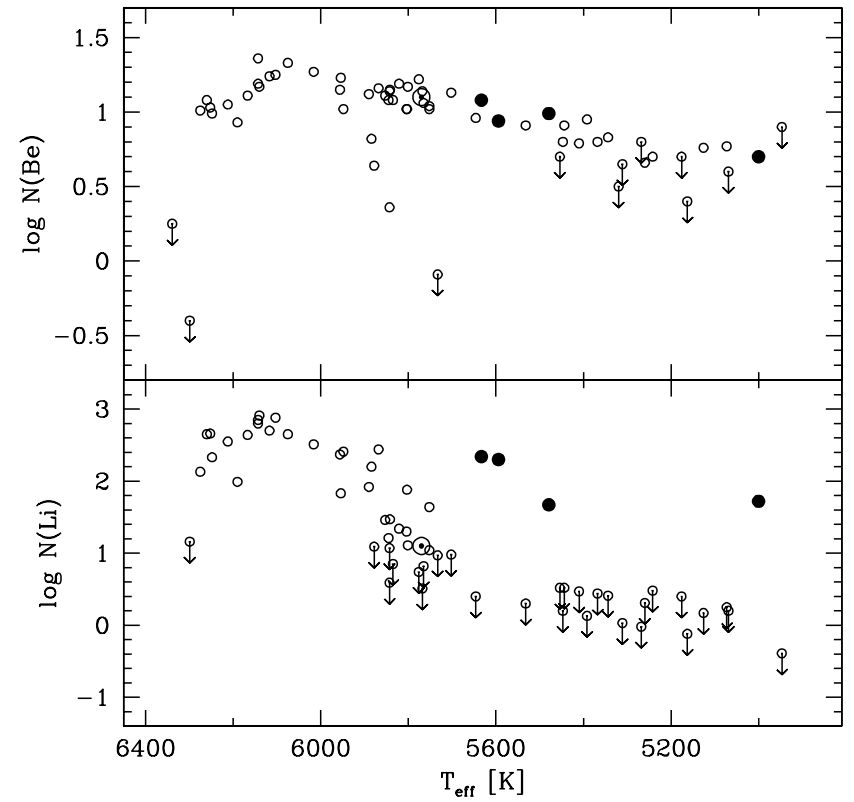

Fig. 11. Beryllium (upper panel) and Li (lower panel) abundances as a function of effective temperature for the dwarfs in our sample. The 4 dwarfs with effective temperatures below $5700 \mathrm{~K}$ that fall outside of the trend in the Li vs. $T_{\text {eff }}$ plot are denoted by the filled circles.

material into the convective envelope of this star. At this moment the results are not conclusive, and the star remains enigmatic.

\section{6. "Li-rich" dwarfs and simultaneous Li and Be depletion}

In Fig. 11 there are 4 dwarfs (HD 36435, HD 43162, HD 43834, and HD 74576) with effective temperatures below $5700 \mathrm{~K}$, that have measurable Li abundances, and clearly fall off the main trend (see also Fig. 12).

To investigate their status we have tried to derive their ages from measurements of the chromospheric activity $S$ index. Values of $S$ were found in the literature for HD 36435 $(S=0.40), \operatorname{HD} 43834(S=0.18)$, and HD $74576(S=0.74)$ (Henry et al. 1996). For HD 43162 we have derived $S=0.41$, on the Mount-Wilson scale, based on CORALIE spectra using the procedure described in Santos et al. (2000). These values imply ages below 1 Gyr for all the stars (Henry et al. 1996), with the exception of HD 43834.

The $S$ index derived by Henry et al. (1996) for this latter dwarf is based on one single measurement, while from 8 different CORALIE spectra we have derived a higher value of $S=$ 0.33 , a value implying an age below <1 Gyr (for HD 36435 and HD 74576 our CORALIE spectra give $S$ values of 0.49 and 0.69 , respectively, compatible with the results derived by Henry et al. 1996). However, doubt is also cast on the young status of HD 43834 as a young star by its X-ray luminosity $\left(L_{\mathrm{x}}=2.8 \times 10^{27} \mathrm{erg} \mathrm{s}^{-1}-\right.$ Huensch et al. 1998), a value not characteristic of a young object.

A look at Table 1 also reveals that the other three stars have projected rotational velocities above $3.5 \mathrm{~km} \mathrm{~s}^{-1}$. In other words, except perhaps for HD 43834, these objects seem to be quite young, which probably justifies their higher than average Li contents.

HD 43834 thus probably remains the only problematic case. If it is an "old" star, how can we explain that at $\sim 5600 \mathrm{~K}$ it still has some $\mathrm{Li}$ in its atmosphere, clearly failing to follow the trend of the remaining dwarfs of its temperature? A possible explanation would be that this star has recently engulfed planetary (Li-rich, and H-poor) material. However, at this moment we do not have any possibility of confirming this. In any case, this star is not known to be orbited by any planetary-mass companion (see Paper I). Another possible (but probably less likely) explanation would be that the interstellar material from which this star was formed was particularly Li-rich.

Curiously, inspection of Fig. 11 and Table 1 tells us that all these stars already seem to have depleted some of their Be, while there is a slight impression that they may lie near the upper envelope of the $\log N(\mathrm{Be})$ vs. $T_{\text {eff }}$ trend (i.e. these stars may have burned less Be than their older counterparts). This seems to tell us that Be depletion in late-type dwarfs stars starts at early ages (i.e. before $1 \mathrm{Gyr}$ ).

These four stars are thus good examples of cool dwarfs that have burned Be but still have large detectable quantities of $\mathrm{Li}$ in their atmospheres. A curious case among these objects is HD 74576, the coolest and probably youngest star (based on its chromospheric activity) of the four. If its $\mathrm{Li}$ and Be contents are confirmed, $\mathrm{Li}$ is depleted by a factor of $\sim 40$, while $\mathrm{Be}$ is depleted by a factor of $\sim 4$, considering initial values for $\mathrm{Li}$ and $\mathrm{Be}$ of 3.31 and $1.26 \mathrm{dex}$, respectively. The process responsible for this simultaneous $\mathrm{Li}$ and Be depletion might be similar to the one found for F dwarfs (e.g., Deliyannis et al. 1997) - see Sect. 7.

\section{Lithium vs. beryllium}

In Fig. 13 we plot the Li and Be abundances against each other. For simplicity, only dwarfs were considered in this plot. The figure tells us that, overall, there seems to be a correlation between the depletions of $\mathrm{Li}$ and $\mathrm{Be}$, in the sense that stars that have depleted their Be have also strongly depleted their Li. This trend was indeed already clear from e.g. Fig. 11.

In Fig. 13 dwarfs in different temperature regimes are denoted by different symbols. Triangles represent stars with $T_{\text {eff }}>6200 \mathrm{~K}$, open circles stars with $6000<T_{\text {eff }} \leq 6200 \mathrm{~K}$, dots objects with $5600<T_{\text {eff }} \leq 6000 \mathrm{~K}$, and crosses denote stars with $T_{\text {eff }} \leq 5600 \mathrm{~K}$.

Excluding the first group of objects, which lies near the $\mathrm{Li}$ and $\mathrm{Be}$ gap for $\mathrm{F}$ stars and is clearly depleted in both $\mathrm{Li}$ and $\mathrm{Be}$, we can see a clear progression in the $\mathrm{Li} / \mathrm{Be}$ ratios as a function of temperature. For stars with $6000<T_{\text {eff }} \leq 6200 \mathrm{~K}$, depletion of Be has not occurred, while $\mathrm{Li}$ is moderately depleted (the particularly high Be abundances for these stars might be also due to Galactic evolution effects, since these objects are on average quite metal-rich - see Sect. 3). Interestingly, there is some hint of a correlation between $\mathrm{Li}$ and $\mathrm{Be}$ abundances for objects with temperatures above $\sim 6000 \mathrm{~K}-$ see also e.g. Fig. 11 . In the temperature regime between 5600 and $6000 \mathrm{~K}$, we see a large dispersion in the $\mathrm{Li}$ abundances, while the $\mathrm{Be}$ abundances remain very close to the primordial value. On the other hand, 


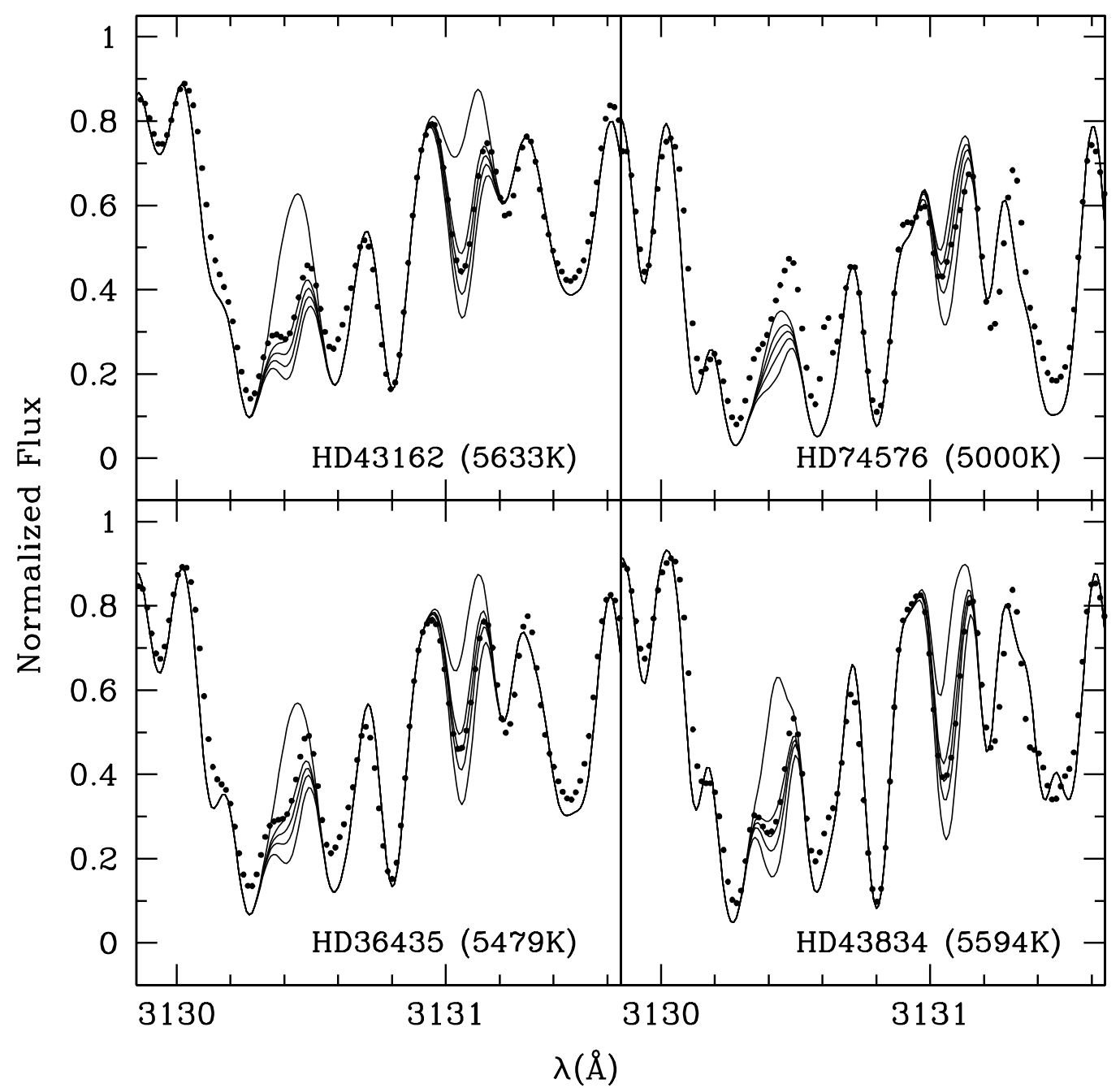

Fig. 12. Spectral syntheses (continuous lines) and observed spectra (points) in the Be II line region for the four stars discussed in Sect. 6. In all panels, the upper and lower syntheses were done with a $\log N(\mathrm{Be})$ of 1.42 (meteoritic) and -10.0 (essentially no Be), respectively. Except for HD 74576, the three other spectral syntheses correspond to the optimal fit and to fits with abundance variations of \pm 0.15 dex. For HD 74576 , these three intermediate fits correspond to the optimal fit and to fits with abundance variations of \pm 0.30 dex.

for temperatures below $5600 \mathrm{~K}$, both $\mathrm{Li}$ and Be are clearly depleted. This temperature seems to mark the onset of strong Be depletion in solar-type stars. Overall, and as already discussed in Santos et al. (2002a), the plot of Fig. 13 gives us information about the temperatures for which $\mathrm{Li}$ and $\mathrm{Be}$ depletion starts.

Although almost all the stars agree with this picture, a few deserve particular comment. The three dwarfs discussed in Sect. 4, all in the temperature range between 5600 and $5900 \mathrm{~K}-$ filled circles - (HD 4391, HD 20766, HD 20807, and maybe also HD 114762), all fall clearly off the general trend in Fig. 13. This result seems to indicate that some kind of non-standard Be and $\mathrm{Li}$ burning mechanism has occurred for these dwarfs and is responsible for the observed Be-gap for solar temperature stars.

Three other objects, in the temperature regime below $5600 \mathrm{~K}$ (crosses), also seem to be particularly Li-rich. These stars, HD 36435, HD 43834, HD 74576, were already discussed in Sect. 6. Except maybe for HD 43834, they are probably young stars, and as mentioned in Sect. 6 their abnormally high Li abundances are probably explained by their young ages. However, Fig. 13 also implies that Be depletion has already occurred for these dwarfs, and is particularly severe for the case of HD 74576 (the coolest of the stars discussed in Sect. 6). This result strongly supports the conclusion that for very cool solar-type dwarfs, Be depletion occurs at high rates early during their lifetimes.

Interestingly, the position of HD 36435, HD 43834 and HD 74576 in the Li vs. Be diagram (the three crosses with the highest Li abundances in Fig. 13) are reasonably well aligned with the points representing stars with $T_{\text {eff }}>6000 \mathrm{~K}$ (open circles and triangles in the same figure). Although preliminary and based on three stars only, this might suggest that a similar mechanism may have been responsible for the depletion of $\mathrm{Li}$ and $\mathrm{Be}$ in both groups of stars.

\section{Concluding remarks}

In this paper we have analyzed the $\mathrm{Be}$ abundances for a large sample of field solar-type stars with temperatures in the range $\sim 4800-6300 \mathrm{~K}$. This sample gives us the possibility to 


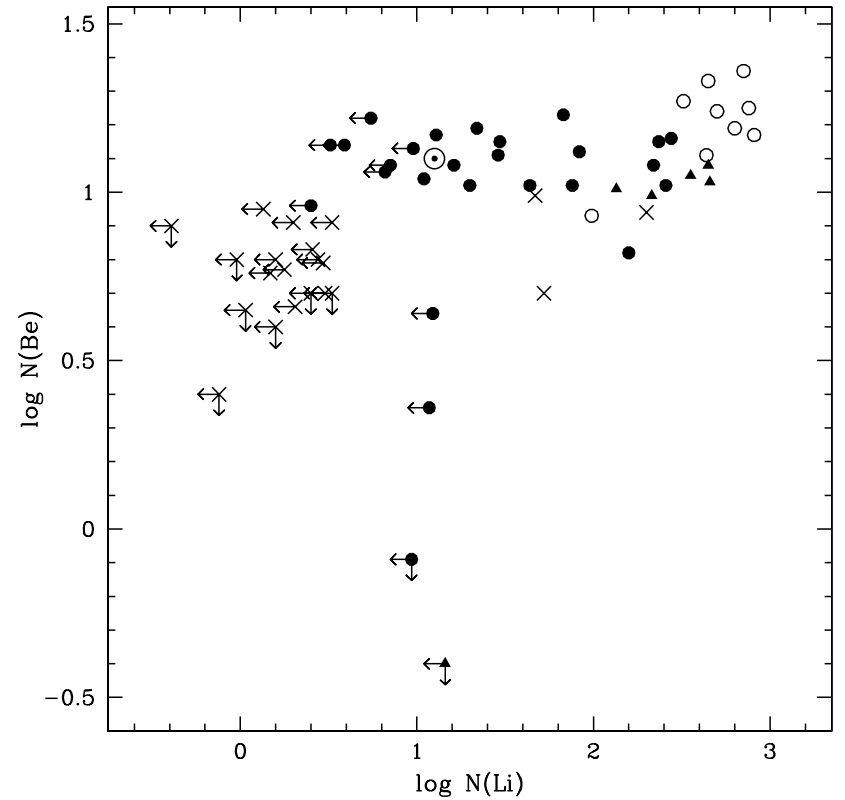

Fig. 13. $\mathrm{Li}$ and Be abundances plotted against each other. Only dwarfs are considered in this plot. Triangles represent stars with $T_{\text {eff }}>$ $6200 \mathrm{~K}$, open circles stars with $6000<T_{\text {eff }} \leq 6200 \mathrm{~K}$, dots objects with $5600<T_{\text {eff }} \leq 6000 \mathrm{~K}$, and crosses stars with $T_{\text {eff }} \leq 5600 \mathrm{~K}$.

study the depletion of Be for dwarfs and sub-giants of different temperature. The major results obtained can be summarized as follows.

- A plot of Be as a function of $T_{\text {eff }}$ shows that the abundances of this light element have a maximum near $6100 \mathrm{~K}$, decreasing both towards higher temperatures (towards the already known Be-gap for F stars), and lower temperatures. This latter trend (see also Santos et al. 2002a), similar to the one found for $\mathrm{Li}$ (that also peak near $6100 \mathrm{~K}$ ), is particularly evident for temperatures below $5600 \mathrm{~K}$. We have explored several possible biases that could lead to the observed behaviour, and found that none could satisfactorily explain it. We thus conclude that this result probably reflects a disagreement between the observations and the models dealing with Be depletion including rotational mixing mechanisms (e.g., Pinsonneault et al. 1990; Deliyannis \& Pinsonneault 1993). A solution may involve the inclusion of internal wave-driven mixing mechanisms in the models (e.g., Montalban \& Schatzman 2000). The fact that literature data for relatively young clusters do not seem do present the same clear trend might indicate that stars cooler than this value do deplete Be during their main-sequence evolution.

- We have found 4 dwarfs (HD 4391, HD 20766, HD 20807, and HD 114762) and one sub-giant star (HD 38529), with temperatures between 5600 and $5900 \mathrm{~K}$, that present particularly low $\mathrm{Be}$ abundances, indicating the presence of some kind of a Be-gap for solar-temperature stars. While for HD 114762 this result can be explained as due to Be Galactic evolution effects (this star is reasonably metalpoor), for the other objects the low Be-abundances imply that some unknown extra-mixing process has occurred.
This result is not predicted by any current Be depletion model.

- A cool sub-giant (HD 23249) star was discovered for which only an upper limit for the Be abundance was measured, but that presents clearly detectable amount of $\mathrm{Li}$ in its atmosphere. We have no clear explanation for this, although some possibilities, like the accretion of planets, or the dredge-up of Be from a "buffer", may account for the observed $\mathrm{Li}$ and Be contents. Other sub-giants in our sample present "normal" Be abundances (they follow the general trend observed for dwarfs), although two of them (HD 10697 and HD 117176) also have particularly high $\mathrm{Li}$ values.

- We have found four dwarfs with temperatures below $5700 \mathrm{~K}$ with particularly high $\mathrm{Li}$ abundances, all presenting signs of $\mathrm{Be}$ depletion. At least three of these (HD 36435, HD 43162, and HD 74576) are young, while another (HD 43834) is probably older than 1 Gyr, making its $\mathrm{Li}$ and $\mathrm{Be}$ content quite difficult to explain. For the three younger dwarfs, the "depleted" Be pattern suggests that the depletion of this light element occurs at early ages in the coolest solar-type dwarfs (this is particularly evident for HD 74576). A comparison of $\mathrm{Li}$ and $\mathrm{Be}$ abundances also suggests that the mechanism responsible for this depletion may be similar to the one found for the hotter ( $T_{\text {eff }}$ above $\sim 6100 \mathrm{~K}$ ) F-dwarfs.

- A comparison of $\mathrm{Li}$ and Be suggests that their abundances follow a clear progression. For stars with temperatures above $\sim 6200 \mathrm{~K}$, these light elements are both depleted, in the well known $\mathrm{Li}$ and Be-gaps for F stars. A maximum in the abundances of $\mathrm{Li}$ and $\mathrm{Be}$ is found for temperatures between 6000 and $6200 \mathrm{~K}$, although for Be this maximum may be due to stellar population bias in our sample. For cooler dwarfs $\mathrm{Li}$ is severely depleted, while Be may present a plateau down to a temperature of $\sim 5600 \mathrm{~K}$ that marks the onset of severe Be depletion.

These results seem to defy the current models of Be depletion in solar-type stars. In this respect, of particular interest is the discovery of a class of severely Be-depleted solartype stars, that form some kind of Be-gap, and the suggestion that Be burning does occur for dwarfs with temperatures below $\sim 5600 \mathrm{~K}$. Although preliminary, our data also suggests that Be depletion may occur before or on the main sequence for stars in this temperature regime. To better understand the observed discrepancies it would be important to enlarge our samples, and to obtain precise boron abundances for at least the most problematic cases.

Acknowledgements. We would like to thank the anonymous referee for the careful and constructive report. Support from Fundação para a Ciência e Tecnologia (Portugal) to N.C.S. in the form of a scholarship is gratefully acknowledged.

\section{References}

Allende Prieto, C., \& Lambert, D. L. 2000, AJ, 119, 2445

Anders, R., \& Grevesse, N. 1989, Geochim. Cosmochim. Acta, 53, 197 
Balachandram, S. C., \& Bell, R. A. 1998, Nature, 393, 791

Barnes, S. A. 2003, ApJ, 586, 464

Boesgaard, A. M., \& King, J. R. 1993, AJ, 106, 2309

Boesgaard, A. M., \& King, J. R. 2002, ApJ, 565, 587

Boesgaard, A. M., Deliyannis, C. P., King, J. R., et al. 1999, AJ, 117, 1549

Boesgaard, A. M., Armengaud, E., \& King, J. R. 2003a, ApJ, 582, 410

Boesgaard, A. M., Armengaud, E., \& King, J. R. 2003b, ApJ, 583, 955

Chen, Y. Q., Nissen, P. E., Benoni, T., \& Zhao, G. 2001, A\&A, 371, 943

Chmielewski, Y., Müller, E. A., \& Brault, J. W. 1975, A\&A, 42, 37

Da Silva, L., \& Foy, R. 1987, A\&A, 177, 204

Deliyannis, C. P., \& Pinsonneault, M. H. 1993, in Inside the Stars, ed.

W. W. Weiss, IAU Col., 137, ASP Conf. Ser., 40, 174

Deliyannis, C. P., \& Pinsonneault, M. H. 1997, ApJ, 488, 836

Deliyannis, C. P., Demarque, P., \& Kalawer, S. 1990, ApJS, 73, 21

Donahue, R. 1993, Ph.D. Thesis, New Mexico State University

ESA 1997, The Hipparcos and Tycho catalogue, ESA-SP 1200

García López, R. J., \& Pérez de Taoro, M. R. 1998, A\&A, 334, 599

García López, R. J., Rebolo, R., \& Martín, E. L. 1994, A\&A, 282, 518

García López, R. J., Rebolo, R., \& Pérez de Taoro, M. R. 1995, A\&A, 302,184

Gonzalez, G. 1997, MNRAS, 285, 403

Gonzalez, G. 1998, A\&A, 334, 221

Gonzalez, G., Laws, C., Tyagi, S., \& Reddy, B. E. 2001, AJ, 121, 432

Grevesse, N., \& Sauval, A. J. 1998, Space Sci. Rev., 85, 161

Henry, T. J., Soderblom, D. R., Donahue, R. A., \& Baliunas, S. L. 1996, AJ, 111, 439

Huensch, M., Schmitt, J. H. M. M., \& Voges, W. 1998, A\&AS, 132, 155

Israelian, G., Santos, N. C., Mayor, M., \& Rebolo, R. 2001, Nature, 411, 163

Israelian, G., Santos, N. C., Mayor, M., \& Rebolo, R. 2004, A\&A, 414, 601

Jones, B. F., Fisher, D., \& Soderblom, D. R. 1999, AJ, 117, 330

Kurucz, R. L. 1993, CD-ROMs, ATLAS9 Stellar Atmospheres Programs and $2 \mathrm{~km} \mathrm{~s}^{-1}$ Grid (Cambridge: Smithsonian Astrophys. Obs.)

Kurucz, R. L., Furenlid, I., Brault, J., \& Testerman, L. 1984, Solar Flux Atlas from 296 to 1300 nm, NOAO Atlas No. 1

Molaro, P., Bonifacio, P., Castelli, F., \& Pasquini, L. 1997, A\&A, 319, 593
Montalban, J., \& Schatzman, E. 2000, A\&A, 354, 943

Murray, N., Chaboyer, B., Arras, P., Hansen, B., \& Noyes, R. W. 2001, ApJ, 555, 801

Naef, D., Mayor, M., Beuzit, J. L., et al. 2004, A\&A, 414, 351

Noyes, R. W., Hartmann, L. W., Baliunas, S. L., Duncan, D. K, \& Vaughan, A. H. 1984, ApJ, 279, 763

Pasquini, L., Liu, Q., \& Pallavicini, R. 1994, A\&A, 287, 191

Pasquini, L., Randich, S., \& Pallavicini, R. 1997, A\&A, 325, 535

Pinsonneault, M. H., Kawaler, S. D., \& Demarque, P. 1990, ApJS, 74, 501

Primas, F., Duncan, D. K., \& Thornburn, J. A. 1998, ApJ, 506, L51

Quillen, A. C. 2002, AJ, 124, 400

Ramaty, R., Kozlovsky, B., Lingenfelter, R. E., \& Reeves, H. 1997, ApJ, 488, 730

Randich, S., Sestito, P., \& Pallavicini, R. 2003, A\&A, 399, 133

Randich, S., Gratton, R., Pallavicini, R., Pasquini, L., \& Carretta, E. 1999, A\&A, 348, 487

Randich, S., Martín, E. L., García López, R. J., \& Pallavicini, R. 1998, A\&A, 333, 591

Rebolo, R., Molaro, P., Abia, C., \& Beckman, J. E. 1988, A\&A, 193, 193

Reeves, H. 1994, Rev. Mod. Phys., 66, 193

Rocha-Pinto, H. J., Castilho, B. V., \& Maciel, W. J. 2002, A\&A, 384, 912

Santos, N. C., Mayor, M., Naef, D., et al. 2000, A\&A, 361, 265

Santos, N. C., García López, R. J., Israelian, G., et al. 2002a, A\&A, 386, 1028

Santos, N. C., Mayor, M., Naef, D., et al. 2002b, A\&A, 392, 215

Santos, N. C., Israelian, G., García López, R. J., et al. 2004a, A\&A, submitted (Paper I)

Santos, N. C., Israelian, G., \& Mayor, M. 2004b, A\&A, 15, 1153

Sneden, C. 1973, Ph.D. Thesis, University of Texas

Soderblom, D. R. 1982, ApJ, 263, 269

Soderblom, D. R., Jones, B. F., Balachandran, S., et al. 1993, AJ, 106, 1059

Stephens, A., Boesgaard, A. M., King, J. R., \& Deliyannis, C. P. 1997, ApJ, 491, 339

Swenson, F. J., Faulkner, J., Iglesias, C. A., Rogers, F. J., \& Alexander, D. R. 1994, ApJ, 422, L79

Vaughan, A. H., Preston, G. W., \& Wilson, O. C. 1978, PASP, 90, 267 